\title{
Cambodia: Statistical Appendix
}

This Statistical Appendix report on Cambodia was prepared by a staff team of the International Monetary Fund as background documentation for the periodic consultation with this member country. As such, the views expressed in this document are those of the staff team and do not necessarily reflect the views of the Government of Cambodia or the Executive Board of the IMF.

\footnotetext{
Copies of this report are available to the public from

International Monetary Fund - Publication Services 700 19th Street, N.W. • Washington, D.C. 20431

Telephone: (202) 623-7430 - Telefax: (202) 623-7201

Telex (RCA): 248331 IMF UR

E-mail: publications@imf.org

Internet: http://www.imf.org

Price: $\$ 15.00$ a copy
}

\section{International Monetary Fund Washington, D.C.}




\title{
INTERNATIONAL-MONETARY FUND
}

\author{
CAMBODIA \\ Statistical Appendix \\ Prepared by Kotaro Ishi, Hong Liang and Yan Sun (APD), \\ and Atsushi Masuda (PDR) \\ Approved by the Asia and Pacific Department
}

August 31,2000

Contents

Page

Tables

1. Gross Domestic Product by Sector at Current Prices, 1994-99 ..............................3

2. Gross Domestic Product by Sector at Constant 1993 Prices, 1994-99 _...................4

3. Gross Domestic Product by Expenditure at Current Prices, 1994-99 ....................5

4. $\quad$ Gross Domestic Product by Expenditure at Constant 1993 Prices, 1994-99 ..........6

5. Deflators for Gross Domestic Product by Sector, 1994-99 ……………................

6. Agriculture, Livestock, and Fishery Production, 1994-99 ......................................

7. Agriculture, Livestock, and Fishery Gross Value Added, 1996-99........................9

8. Consumer Price Index, 1996-2000 ............................................................... 10

9. Central Government Operations, 1996-2000 …..............................................11

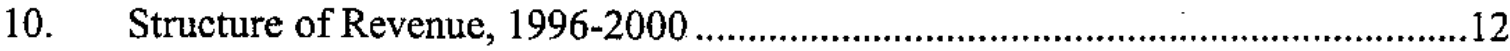

11. Budgetary Expenditure by Economic Classification, 1996-2000 …......................14

12. Budgetary Expenditure by Ministry, 1998-2000 ................................................15

13. Official External Assistance to the Budget, 1996-2000 ………….........................16

14. Profile of the Commercial Bank System ..............................................................17

15. Monetary Survey, 1997-2000 ......................................................................18

16. Sources of Broad Money Growth, 1997-2000 _.................................................19

17. Deposit Money Bank's Balance Sheet, 1997-2000 ….........................................20

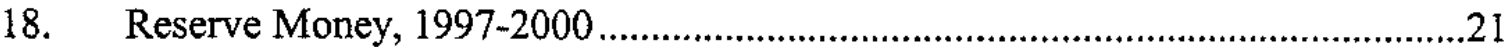

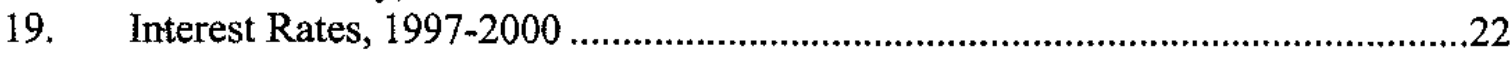

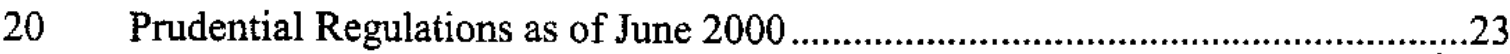

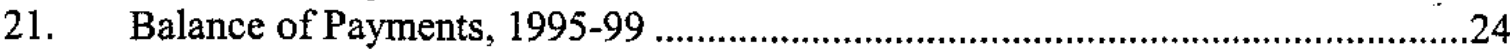

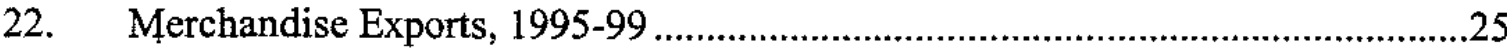

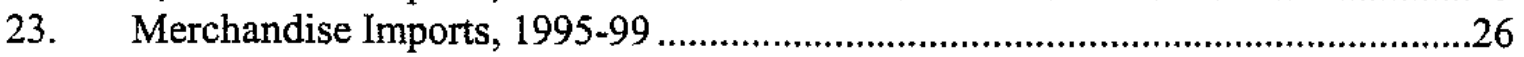

24. Investment Approvals by Sector, 1995-99 ………............................................2.

25. Investment Approvals by Investor Country of Origin, 1995-99.............................28

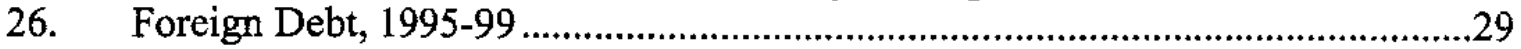

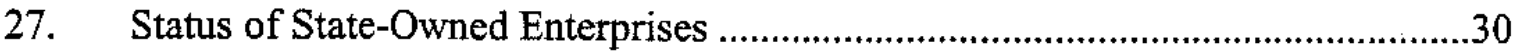


28. Previously State-Owned Enterprises and Assets Sold to the Private Sector. 31

29. State-Owned Enterprises Leased to the Private Sector.........................................32

30 Proposed Privatization Decisions ....................................................................33

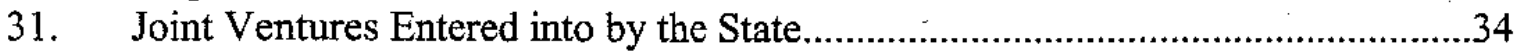

Annex

1. Summary of the Cambodian Tax System ..... 


$$
-3-
$$

Table 1. Cambodia: Gross Domestic Product by Sector at Current Prices, 1994-99

\begin{tabular}{|c|c|c|c|c|c|c|}
\hline & $\overline{1994}$ & 1995 & 1996 & 1997 & 1998 & $\begin{array}{r}1999 \\
\text { Est. }\end{array}$ \\
\hline & \multicolumn{6}{|c|}{ (In billions of riels at current prices) } \\
\hline Agriculture & 2,614 & 3,453 & 3,471 & 3,857 & 4,414 & 4,539 \\
\hline Crops & 1,146 & 2,004 & 1,965 & 2,063 & 2,386 & 2,377 \\
\hline Livestock and poultry & 483 & 478 & 551 & 569 & 683 & 798 \\
\hline Fisheries & 464 & 471 & 555 & 592 & 726 & 933 \\
\hline Forestry and logging & 522 & 500 & 400 & 633 & 619 & 430 \\
\hline Industry & 806 & 986 & 1,212 & 1,460 & 1,828 & 2,156 \\
\hline Mining & 11 & 12 & 13 & 14 & 15 & 19 \\
\hline Manufacturing & 522 & 580 & 754 & 989 & 1,352 & 1,501 \\
\hline Food, beverages, and tobacco & 220 & 249 & 299 & 330 & 390 & 420 \\
\hline Textile, wearing apparel, and footwear & so & 69 & 132 & 305 & 507 & 718 \\
\hline Wood, paper, and publishing & 96 & 83 & 113 & 148 & 230 & 92 \\
\hline Rubber manufacturing & 20 & 31 & 45 & 33 & 25 & 35 \\
\hline Other manufacturing & 135 & 148 & 165 & 174 & 201 & 236 \\
\hline Electricity, gas, and water & 31 & 40 & 40 & 41 & 47 & 43 \\
\hline Construction & 242 & 355 & 406 & 416 & 414 & 593 \\
\hline Services & 2,463 & 2,781 & 3,221 & 3,419 & 3,835 & 4,279 \\
\hline Trade & 869 & 973 & 1,078 & 1,149 & 1,241 & 1,332 \\
\hline Hotels and restaurants & 237 & 285 & 350 & 375 & 418 & 524 \\
\hline Transport and communications & 386 & 423 & 510 & 534 & 587 & 708 \\
\hline Finance & 27 & 77 & 87 & 98 & 100 & 110 \\
\hline Public administration & 222 & 234 & 296 & 305 & 311 & 380 \\
\hline Real estate and business & 452 & 483 & 537 & 601 & 732 & 764 \\
\hline Other services & 271 & 307 & 362 & 357 & 446 & 461 \\
\hline Taxes on products less subsidies & 334 & 395 & 483 & 537 & 579 & 638 \\
\hline Less: imputed bank charges & 17 & 73 & 63 & 123 & 124 & 142 \\
\hline \multirow[t]{2}{*}{ Gross domestic product (GDP) } & 6,201 & 7,543 & 8,325 & 9,149 & 10,531 & 11,470 \\
\hline & \multicolumn{6}{|c|}{ (Percentage change from previous period) } \\
\hline Agriculture & 2.3 & 32.1 & 0.5 & 11.1 & 14.4 & 2.8 \\
\hline Crops & 1.2 & 74.9 & -1.9 & 5.0 & 15.6 & -0.3 \\
\hline Livestock and poultry & -8.8 & -1.0 & 15.4 & 3.3 & 19.9 & 17.0 \\
\hline Fisheries & -24.0 & 1.6 & 17.7 & 6.7 & 22.7 & 28.5 \\
\hline Forestry and logging & 83.1 & -4.2 & -20.0 & 58.3 & -2.1 & -30.5 \\
\hline Industry & 6.6 & 22.3 & 22.9 & 20.4 & 25.2 & 18.0 \\
\hline Mining & 1.0 & 4.2 & 11.4 & 7.1 & 10.7 & 23.1 \\
\hline Manufacturing & 6.7 & 11.1 & 29.9 & 31.3 & 36.7 & 11.0 \\
\hline Of which: Textile, wearing apparel, and footwear & -29.0 & 37.0 & 91.9 & 130.3 & 66.4 & 41.6 \\
\hline Electricity, gas, and water & 28.0 & 28.1 & 1.5 & 1.4 & 14.5 & -6.6 \\
\hline Construction & 4.5 & 46.7 & 14.2 & 2.5 & -0.5 & 43.3 \\
\hline Services & -5.0 & 12.9 & 15.8 & 6.1 & 12.2 & 11.6 \\
\hline Trade & -8.7 & 11.9 & 10.9 & 6.5 & 8.0 & 7.4 \\
\hline Hotels and restaurants & 21.1 & 20.4 & 23.0 & 7.1 & 11.5 & 25.3 \\
\hline Transport and communications & 5.0 & 9.7 & 20.5 & 4.8 & 9.8 & 20.6 \\
\hline Finance & 15.0 & 182.1 & 13.1 & 13.0 & 2.6 & 9.8 \\
\hline Public administration & 58.3 & 5.2 & 26.7 & 3.0 & 1.9 & 22.3 \\
\hline Real estate and business & -27.4 & 7.1 & 11.1 & 11.8 & 21.9 & 4.3 \\
\hline Other services & -7.0 & 13.3 & 18.2 & -1.5 & 24.8 & 3.3 \\
\hline Gross domestic product (GDP) & 1.9 & 21.6 & 10.4 & 9.9 & 15.1 & 8.9 \\
\hline
\end{tabular}

Sources: National Institute of Statistics, Ministry of Planning. 


$$
-4-
$$

Table 2. Cambodia: Gross Domestic Product by Sector at Constant 1993 Prices, 1994-99

\begin{tabular}{|c|c|c|c|c|c|c|}
\hline & 1994 & 1995 & 1996 & 1997 & 1998 & $\begin{array}{r}1999 \\
\text { Est. }\end{array}$ \\
\hline . & \multicolumn{6}{|c|}{ (In billions of riels at constant 1993 prices) } \\
\hline Agriculture & 2,616 & 2,812 & 2,874 & 3,039 & 3,115 & 3,162 \\
\hline Crops & 1,015 & 1,301 & 1,358 & 1,321 & 1,329 & 1,450 \\
\hline Livestock and poultry & 527 & 542 & 555 & 561 & 593 & 564 \\
\hline Fisheries & 574 & 582 & 630 & 679 & 716 & 830 \\
\hline Forestry and logging & 501 & 388 & 331 & 478 & 477 & 319 \\
\hline Industry & 788 & 947 & 1,058 & 1,274 & 1,383 & 1,541 \\
\hline Mining & 12 & 12 & 12 & 12 & 12 & 13 \\
\hline Manufacturing & 513 & 569 & 675 & 912 & 1,061 & 1,158 \\
\hline Food, beverages, and tobacco & 223 & 227 & 245 & 249 & 253 & 263 \\
\hline Textile, wearing apparel, and footwear & 57 & 69 & 136 & 309 & 453 & 637 \\
\hline Wood, paper, and publishing & 85 & 105 & 119 & 192 & 183 & 68 \\
\hline Rubber manufacturing & 19 & 25 & 34 & 28 & 29 & 38 \\
\hline Other manufacturing & 129 & 142 & 142 & 133 & 143 & 153 \\
\hline Electricity, gas, and water & 26 & 35 & 36 & 36 & 38 & 39 \\
\hline Construction & 237 & 331 & 335 & 314 & 272 & 331 \\
\hline Services & 2,586 & 2,695 & 2,825 & 2,721 & 2,685 & 2,840 \\
\hline Trade & 925 & 919 & 917 & 895 & 848 & 862 \\
\hline Hotels and restaurants & 243 & 287 & 305 & 295 & 283 & 334 \\
\hline Transport and communications & 404 & 405 & 440 & 377 & 367 & 415 \\
\hline Finance & 29 & 69 & 74 & 76 & 69 & 70 \\
\hline Public administration & 210 & 215 & 252 & 261 & 259 & 269 \\
\hline Real estate and business & 478 & 493 & 497 & 511 & 531 & 548 \\
\hline Other services & 296 & 309 & 340 & 307 & 329 & 342 \\
\hline Taxes on products less subsidies & 352 & 356 & 412 & 433 & 412 & 432 \\
\hline Less: imputed bank charges & 18 & 67 & 54 & 89 & 83 & 92 \\
\hline \multirow[t]{2}{*}{ Gross domestic product (GDP) } & 6,323 & 6,744 & 7,115 & 7,378 & 7,512 & 7,885 \\
\hline & \multicolumn{6}{|c|}{ (Percent change from previous period) } \\
\hline Agriculture & 2.3 & 7.5 & 2.2 & 5.8 & 2.5 & 1.5 \\
\hline Crops & -10.4 & 28.2 & 4.4 & -2.7 & 0.6 & 9.1 \\
\hline Livestock and poultry & -0.5 & 2.8 & 2.5 & 1.2 & 5.6 & -4.9 \\
\hline Fisheries & -5.9 & 1.4 & 8.3 & 7.8 & 5.4 & 16.0 \\
\hline Forestry and logging & 75.7 & -22.5 & -14.7 & 44.3 & -0.1 & -33.2 \\
\hline Industry & 4.2 & 20.2 & 11.7 & 20.4 & 8.6 & 11.4 \\
\hline Mining & 7.2 & 2.8 & -4.6 & 0.1 & 1.4 & 8.0 \\
\hline Manufacturing & 4.9 & 10.8 & 18.7 & 35.1 & 16.4 & 9.1 \\
\hline Of which: Textile, wearing apparel, and & -18.9 & 19.9 & 96.6 & 128.0 & 46.5 & 40.8 \\
\hline Electricity, gas, and water & 7.5 & 37.0 & 0.9 & 1.6 & 3.7 & 3.8 \\
\hline Construction & 2.2 & 39.8 & 1.3 & -6.4 & -13.2 & 21.6 \\
\hline Services & -0.2 & 4.2 & 4.8 & -3.7 & -1.3 & 5.8 \\
\hline Trade & -2.9 & -0.7 & -0.2 & -2.4 & -5.3 & 1.7 \\
\hline Hotels and restaurants & 24.5 & 17.9 & 6.2 & -3.3 & -4.1 & 18.3 \\
\hline Transport and communications & 10.0 & 0.1 & 8.8 & -14.4 & -2.6 & 13.2 \\
\hline Finance & 21.2 & 141.2 & 6.7 & 3.6 & -9.5 & 1.9 \\
\hline Public administration & 49.9 & 2.0 & 17.3 & 3.5 & -0.5 & 3.7 \\
\hline Real estate and business & -23.1 & 3.0 & 0.9 & 2.7 & 3.9 & 3.3 \\
\hline Other services & 1.7 & 4.4 & 10.1 & -9.9 & 7.2 & 3.9 \\
\hline Gross domestic product (GDP) & 3.9 & 6.7 & 5.5 & 3.7 & 1.8 & 5.0 \\
\hline
\end{tabular}


$-5-$

Table 3. Cambodia: Gross Domestic Product by Expenditure at Current Prices, 1994-99

\begin{tabular}{|c|c|c|c|c|c|c|}
\hline & 1994 & 1995 & 1996 & 1997 & 1998 & $\begin{array}{r}1999 \\
\text { Est. }\end{array}$ \\
\hline & \multicolumn{6}{|c|}{ (In billions of riels at current prices) } \\
\hline Household final consumption expenditure & 6,002 & 6,799 & 7,354 & 8,041 & 9,377 & 9,916 \\
\hline Government consumption expenditure & 493 & 413 & 529 & 553 & 590 & 720 \\
\hline Gross fixed capital formation & 700 & 957 & 1,125 & 1,192 & 1,360 & 1,818 \\
\hline Durable equipment & 215 & 263 & 387 & 436 & 608 & 740 \\
\hline Construction & 484 & 693 & 737 & 756 & 752 & 1,078 \\
\hline Increase in stocks & 57 & 18 & 144 & 122 & -98 & 288 \\
\hline Exports of goods and services & 1,412 & 2,407 & 2,137 & 3,048 & 3,785 & 4,264 \\
\hline Merchandise, f.o.b. & 1,272 & 2,124 & 1,713 & 2,605 & 3,371 & 3,774 \\
\hline Services & 141 & 283 & 425 & 443 & 414 & 490 \\
\hline Less: Imports of goods and services & 2,384 & 3,589 & 3,597 & 4,067 & 4,864 & 5,632 \\
\hline Merchandise, c.i.f. & 2,026 & 3,124 & 3,033 & 3,479 & 4,203 & 4,875 \\
\hline Services & 359 & 465 & 564 & 588 & 662 & 757 \\
\hline Statistical discrepancy & -79 & 538 & 633 & 260 & 380 & 96 \\
\hline \multirow[t]{2}{*}{ Gross domestic expenditures } & 6,201 & 7,543 & 8,325 & 9,149 & 10,531 & 11,470 \\
\hline & \multicolumn{6}{|c|}{ (Share as a percent of GDP) } \\
\hline Household final consumption expenditure & 96.8 & 90.1 & 88.3 & 87.9 & 89.0 & 86.4 \\
\hline Government consumption expenditure & 7.9 & 5.5 & 6.4 & 6.0 & 5.6 & 6.3 \\
\hline Gross fixed capital formation & 11.3 & 12.7 & 13.5 & 13.0 & 12.9 & 15.8 \\
\hline Durable equipment & 3.5 & 3.5 & 4.6 & 4.8 & 5.8 & 6.5 \\
\hline Construction & 7.8 & 9.2 & 8.9 & 8.3 & 7.1 & 9.4 \\
\hline Increase in stocks & 0.9 & 0.2 & 1.7 & 1.3 & -0.9 & 2.5 \\
\hline Exports of goods and services & 22.8 & 31.9 & 25.7 & 33.3 & 35.9 & 37.2 \\
\hline Merchandise, f.o.b. & 20.5 & 28.2 & 20.6 & 28.5 & 32.0 & 32.9 \\
\hline Services & 2.3 & 3.8 & 5.1 & 4.8 & 3.9 & 4.3 \\
\hline Less: Imports of goods and services & 38.4 & 47.6 & 43.2 & 44.5 & 46.2 & 49.1 \\
\hline Merchandise, c.i.f. & 32.7 & 41.4 & 36.4 & 38.0 & 39.9 & 42.5 \\
\hline Services & 5.8 & 6.2 & 6.8 & 6.4 & 6.3 & 6.6 \\
\hline Statistical discrepancy & -1.3 & 7.1 & 7.6 & 2.8 & 3.6 & 0.8 \\
\hline Gross domestic expenditures & 100.0 & 100.0 & 100.0 & 100.0 & 100.0 & 100.0 \\
\hline
\end{tabular}

Sources: National Institute of Statistics, Ministry of Planning. 
$-6-$

Table 4. Cambodia: Gross Domestic Product by Expenditure at Constant 1993 Prices, 1994-99

\begin{tabular}{|c|c|c|c|c|c|c|}
\hline & 1994 & 1995 & 1996 & 1997 & 1998 & $\begin{array}{r}1999 \\
\text { Est. }\end{array}$ \\
\hline & \multicolumn{6}{|c|}{ (In billions of riels at constant prices) } \\
\hline Household final consumption expenditure & 6,194 & 6,464 & 6,542 & 6,547 & 6,651 & 6,764 \\
\hline Government consumption expenditure & 467 & 380 & 450 & 431 & 395 & 434 \\
\hline Gross fixed capital formation & 633 & 841 & 968 & 973 & 1,107 & 1,292 \\
\hline Durable equipment & 159 & 195 & 358 & 402 & 612 & 690 \\
\hline Construction & 474 & 646 & 610 & 571 & 495 & 602 \\
\hline Increase in stocks & 61 & 16 & 144 & 115 & -90 & 199 \\
\hline Exports of goods and services & 1,585 & 2,241 & 1,815 & 2,688 & 2,953 & 3,518 \\
\hline Merchandise, f.o.b. & 1,425 & 1,972 & 1,456 & 2,344 & 2,678 & 3,203 \\
\hline Services & 159 & 269 & 359 & 343 & 276 & 315 \\
\hline Less: Imports of goods and services & 2,046 & 3,340 & 2,975 & 3,138 & 3,336 & 4,090 \\
\hline Merchandise, c.i.f. & 1,668 & 2,913 & 2,495 & 2,671 & 2,893 & 3,601 \\
\hline Services & 377 & 427 & 480 & 468 & 443 & 489 \\
\hline Statistical discrepancy & -570 & 142 & 172 & -237 & -169 & -231 \\
\hline \multirow[t]{2}{*}{ Gross domestic expenditures } & 6,323 & 6,744 & 7,115 & 7,378 & 7,512 & 7,885 \\
\hline & \multicolumn{6}{|c|}{ (Percent change from previous period) } \\
\hline Household final consumption expenditure & 3.4 & 4.4 & 1.2 & 0.1 & 1.6 & 1.7 \\
\hline Government consumption expenditure & 52.5 & -18.6 & 18.5 & -4.2 & -8.3 & 9.7 \\
\hline Gross fixed capital formation & 13.4 & 32.9 & 15.1 & 0.5 & 13.7 & 16.7 \\
\hline Durable equipment & 68.1 & 22.5 & 84.1 & 12.3 & 52.0 & 12.8 \\
\hline Construction & 2.2 & 36.4 & -5.7 & -6.4 & -13.2 & 21.6 \\
\hline Increase in stocks & 1134.8 & -73.4 & 782.5 & -19.7 & -177.7 & -321.4 \\
\hline Exports of goods and services & 32.8 & 41.4 & -19.0 & 48.1 & 9.9 & 19.1 \\
\hline Merchandise, f.o.b. & 41.2 & 38.3 & -26.2 & 61.0 & 14.2 & 19.6 \\
\hline Services & -13.4 & 69.3 & 33.3 & -4.5 & -19.6 & 14.3 \\
\hline Less: Imports of goods and services & 23.4 & 63.3 & -10.9 & 5.5 & 6.3 & 22.6 \\
\hline Merchandise, c.i.f. & 32.1 & 74.6 & -14.3 & 7.0 & 8.3 & 24.5 \\
\hline Services & -4.6 & 13.2 & 12.4 & -2.6 & -5.3 & 10.5 \\
\hline Gross domestic expenditures & 3.9 & 6.7 & 5.5 & 3.7 & 1.8 & 5.0 \\
\hline
\end{tabular}

Sources: National Institute of Statistics, Ministry of Planning. 
Table 5. Cambodia: Deflators for Gross Domestic Product by Sector, 1994-99

(Percent change from previous period)

\begin{tabular}{|c|c|c|c|c|c|c|}
\hline & $\overline{1994}$ & 1995 & 1996 & 1997 & 1998 & $\begin{array}{r}1999 \\
\text { Est. }\end{array}$ \\
\hline Agriculture & -0.1 & 22.9 & -1.6 & 5.1 & 11.7 & 1.3 \\
\hline Crops & 13.0 & 36.4 & -6.1 & 7.9 & 14.9 & -8.6 \\
\hline Livestock and poultry & -8.4 & -3.7 & 12.6 & 2.1 & 13.6 & 23.0 \\
\hline Fisheries & -19.2 & 0.2 & 8.6 & -1.0 & 16.5 & 10.8 \\
\hline Forestry and logging & 4.2 & 23.5 & -6.2 & 9.7 & -2.1 & 4.0 \\
\hline Industry & 2.3 & 1.7 & 10.0 & 0.0 & 15.3 & 5.9 \\
\hline Mining & -5.8 & 1.4 & 16.7 & 7.0 & 9.1 & 14.0 \\
\hline Manufacturing & 1.7 & 0.3 & 9.4 & -2.8 & 17.4 & 1.7 \\
\hline Food, beverages, and tobacco & -1.2 & 10.9 & 11.2 & 8.5 & 16.6 & 3.7 \\
\hline Textile, wearing apparel, and footwear & -12.5 & 14.3 & -2.4 & 1.0 & 13.6 & 0.5 \\
\hline Wood, paper, and publishing & 12.9 & -30.2 & 20.2 & -18.9 & 63.3 & 8.5 \\
\hline Rubber manufacturing & 3.3 & 17.9 & 7.1 & -10.4 & -28.0 & 12.3 \\
\hline Other manufacturing & 5.3 & -0.8 & 11.8 & 11.8 & 7.1 & 9.9 \\
\hline Electricity, gas, and water & 19.1 & -6.5 & 0.6 & -0.3 & 10.4 & -10.0 \\
\hline Construction & 2.2 & 4.9 & 12.7 & 9.5 & 14.6 & 17.9 \\
\hline Services & -4.7 & 8.3 & 10.5 & 10.2 & 13.7 & 5.5 \\
\hline Trade & -6.0 & 12.7 & 11.0 & 9.1 & 14.1 & 5.6 \\
\hline Hotels and restaurants & -2.7 & 2.1 & 15.8 & 10.7 & 16.2 & 5.9 \\
\hline Transport and communications & -4.6 & 9.6 & 10.8 & 22.3 & 12.8 & 6.6 \\
\hline Finance & -5.2 & 17.0 & 6.0 & 9.1 & 13.3 & 7.7 \\
\hline Public administration & 5.6 & 3.1 & 8.0 & -0.5 & 2.4 & 18.0 \\
\hline Real estate and business & -5.6 & 3.9 & 10.1 & 8.9 & 17.3 & 0.9 \\
\hline Other services & -8.6 & 8.5 & 7.3 & 9.4 & 16.4 & -0.6 \\
\hline Taxes on products & -4.9 & 16.7 & 5.7 & 5.8 & 13.4 & 5.0 \\
\hline Less: imputed bank charges & -5.2 & 14.8 & 8.0 & 17.6 & 8.0 & 3.5 \\
\hline Gross domestic product (GDP) & -1.9 & 14.0 & 4.6 & 6.0 & 13.1 & 3.8 \\
\hline
\end{tabular}

Sources: National Institute of Statistics, Ministry of Planning. 
Table 6. Cambodia: Agriculture, Livestock, and Fishery Production, 1994-99

\begin{tabular}{|c|c|c|c|c|c|c|c|}
\hline & $\begin{array}{r}\text { Unit } \\
(000)\end{array}$ & 1994 & 1995 & 1996 & 1997 & 1998 & $\begin{array}{l}1999 \\
\text { Est. }\end{array}$ \\
\hline \multicolumn{8}{|l|}{ Agriculture 1/ } \\
\hline Rice & Tons & 2,223 & 3,448 & 3,458 & 3,415 & 3,510 & 4,030 \\
\hline Maize & Tons & 45 & 55 & 65 & 42 & 49 & 69 \\
\hline Cassava & Tons & 65 & 82 & 70 & 77 & 67 & 108 \\
\hline Sweet potato & Tons & 36 & 39 & 38 & 29 & 30 & 30 \\
\hline Vegetables & Tons & 197 & 193 & 250 & 250 & 217 & 215 \\
\hline Mungbean & Tons & 17 & 20 & 14 & 15 & 9 & 14 \\
\hline Peanut & Tons & 5 & 7 & 6 & 7 & 7 & 8 \\
\hline Soybean & Tons & 23 & 17 & 28 & 56 & 28 & 33 \\
\hline Sesame & Tons & 4 & 4 & 5 & 7 & 5 & 8 \\
\hline Sugar cane & Tons & 219 & 202 & 171 & 188 & 133 & 182 \\
\hline Tobacco & Tons & 12 & 11 & 10 & 10 & 10 & 10 \\
\hline Jute & Tons & 2 & 1 & 2 & 2 & 1 & - \\
\hline Rubber & Tons & 31 & 35 & 44 & 32 & 34 & 45 \\
\hline \multicolumn{8}{|l|}{ Livestock } \\
\hline Cows & Heads & 2,622 & 2,778 & 2,762 & 2,872 & 2,680 & 2,887 \\
\hline Buffalos & Heads & 814 & 765 & 744 & 766 & 694 & 696 \\
\hline Pigs & Heads & 2,002 & 2,039 & 2,151 & 2,237 & 2,339 & 2,363 \\
\hline Poultry & Heads & 10,094 & 10,067 & 11,412 & 11,982 & 13,167 & 15,085 \\
\hline \multicolumn{8}{|l|}{ Fishery } \\
\hline Fresh fish and shrimp & Tons & 103 & 113 & 104 & 115 & 122 & 284 \\
\hline Crocodiles & Heads & 6 & 15 & 20 & 17 & 41 & 25 \\
\hline
\end{tabular}

Source: Data provided by the Cambodian authorities.

$1 /$ Harvest year for crops; tons are metric tons. 
Table 7. Cambodia: Agriculture, Livestock, and Fishery Gross Value Added (GVA), 1996-99 (In billions of riels at constant 1993 prices)

\begin{tabular}{lrrrr}
\hline & 1996 & 1997 & 1998 & $\begin{array}{r}1999 \\
\text { Est. }\end{array}$ \\
\hline Agriculture, Fishery, \& Forestry & & & & 3,115 \\
Agricultural Crops & 2,874 & 3,039 & 3,162 \\
Rice & 1,358 & 1,321 & 1,329 & 1,450 \\
Corn & 783 & 773 & 795 & 859 \\
Cassava & 27 & 18 & 21 & 28 \\
Sweet potato & 25 & 28 & 24 & 39 \\
Mungbean & 14 & 10 & 11 & 11 \\
Soybean & 18 & 20 & 12 & 19 \\
Groundnut & 25 & 49 & 24 & 29 \\
Sesame & 09 & 11 & 10 & 12 \\
Vegetables & 06 & 07 & 06 & 09 \\
Sugarcane & 182 & 129 & 158 & 156 \\
Black pepper & 20 & 22 & 16 & 22 \\
Tobacco & 01 & $\ldots$ & $\ldots$ & $\ldots$ \\
Cotton & 18 & 19 & 19 & 19 \\
Jute & $\ldots$ & $\ldots$ & $\ldots$ & $\ldots$ \\
Rubber & 01 & 01 & 01 & 0 \\
Other crops & 60 & 59 & 53 & 60 \\
& 169 & 174 & 180 & 187 \\
Livestock \& Poultry & & & & \\
& 555 & 561 & 593 & 564 \\
Fisheries & & & & \\
& 630 & 679 & 716 & 830 \\
Forestry \& Logging & & & & \\
& 331 & 478 & 477 & 319 \\
\hline
\end{tabular}

Source: Data provided by the Cambodian authorities. 
Table 8. Consumer Price Index, 1996-2000

\begin{tabular}{|c|c|c|c|c|c|c|c|c|c|}
\hline & $\begin{array}{l}\text { CPI ALL } \\
\text { ITEMS }\end{array}$ & $\begin{array}{r}\text { Food, } \\
\text { Beverages } \\
\text { and } \\
\text { Tabacco } \\
\end{array}$ & $\begin{array}{r}\text { Clothing } \\
\text { and } \\
\text { Footwear }\end{array}$ & $\begin{array}{r}\text { Housing } \\
\text { and } \\
\text { Utilities }\end{array}$ & $\begin{array}{r}\text { House } \\
\text { Furnishings } \\
\text { and HH. } \\
\text { Operations }\end{array}$ & $\begin{array}{r}\text { Medical } \\
\text { Care }\end{array}$ & $\begin{array}{r}\text { Transportation } \\
\text { and } \\
\text { Communication }\end{array}$ & $\begin{array}{r}\text { Recreation } \\
\text { and } \\
\text { Education }\end{array}$ & $\begin{array}{r}\text { Personal } \\
\text { Care and } \\
\text { Effects }\end{array}$ \\
\hline \multicolumn{10}{|c|}{ (Index, July-Sep., 1994=100) } \\
\hline Dec-96 & 115.9 & 119.2 & 103.6 & 108.7 & 121.6 & 115.8 & 125.5 & 114.7 & 101.5 \\
\hline Dec-97 & 126.6 & 122.8 & 111.7 & 122.5 & 121.6 & 146.7 & 161.9 & 118.2 & 107.8 \\
\hline Dec-98 & 143.4 & 148.1 & 118.8 & 129.3 & 144.2 & 159.3 & 169.0 & 127.2 & 116.0 \\
\hline $\operatorname{Jan}-99$ & 140.5 & 143.9 & 120.2 & 128.5 & 152.8 & 150.0 & 165.8 & 126.3 & 116.1 \\
\hline Feb-99 & 141.1 & 145.1 & 123.9 & 128.7 & 152.7 & 148.4 & 164.8 & 126.3 & 115.0 \\
\hline Mar-99 & 140.7 & 144.2 & 123.3 & 128.6 & 150.3 & 145.3 & 167.2 & 129.2 & 116.2 \\
\hline Apr-99 & 141.5 & 144.5 & 120.6 & 129.4 & 150.6 & 146.8 & 172.5 & 129.2 & 114.6 \\
\hline May-99 & 143.3 & 148.2 & 120.6 & 129.2 & 151.4 & 148.1 & 172.5 & 129.2 & 113.1 \\
\hline Jun-99 & 144.2 & 151.2 & 120.7 & 129.0 & 150.9 & 142.1 & 171.1 & 124.8 & 114.5 \\
\hline Jul-99 & 144.4 & 151.1 & 120.1 & 130.8 & 165.7 & 138.7 & 169.1 & 124.0 & 111.9 \\
\hline Aug-99 & 143.6 & 149.1 & 118.7 & 130.5 & 165.7 & 139.3 & 172.5 & 124.0 & 114.4 \\
\hline Sep-99 & 144.0 & 149.9 & 118.7 & 129.8 & 166.6 & 140.1 & 173.7 & 121.7 & 115.4 \\
\hline Oct-99 & 143.7 & 149.1 & 117.9 & 129.8 & 165.4 & 143.2 & 173.5 & 121.7 & 113.8 \\
\hline Nov-99 & 143.8 & 149.5 & 115.8 & 129.8 & 164.8 & 143.5 & 173.6 & 121.7 & 113.2 \\
\hline Dec-99 & 142.6 & 147.7 & 115.9 & 129.4 & 164.9 & 140.0 & 174.4 & 119.1 & 114.0 \\
\hline $\operatorname{Jan}-00$ & 141.0 & 143.4 & 116.1 & 132.5 & 159.5 & 140.5 & 173.2 & 118.6 & 113.8 \\
\hline Feb-00 & 140.6 & 141.5 & 118.7 & 132.7 & 160.8 & 140.8 & 177.6 & 118.6 & 112.9 \\
\hline Mar-00 & 140.9 & 141.3 & 119.4 & 132.5 & 161.8 & 137.3 & 184.4 & 118.8 & 113.4 \\
\hline Apr -00 & 140.3 & 140.5 & 121.0 & 132.7 & 161.1 & 138.1 & 178.9 & 119.7 & 115.1 \\
\hline May-00 & 139.5 & 139.1 & 121.4 & 133.2 & 161.1 & 139.0 & 176.5 & 119.7 & 112.4 \\
\hline Jun-00 & 139.4 & 139.7 & 115.1 & 132.6 & 160.3 & 134.6 & 179.3 & 118.1 & 116.5 \\
\hline \multicolumn{10}{|c|}{ (12 Monthly Percentage Change) } \\
\hline Dec-96 & 10.0 & 11.6 & 3.6 & 10.5 & 15.2 & 4.5 & 0.4 & 2.3 & 2.7 \\
\hline Dec-97 & 9.2 & 3.0 & 7.9 & 12.7 & 0.0 & 26.7 & 29.0 & 3.0 & 6.2 \\
\hline Dec-98 & 13.3 & 20.6 & 6.3 & 5.6 & 18.5 & 8.6 & 4.4 & 7.6 & 7.6 \\
\hline Jun-99 & 4.9 & 10.5 & 3.0 & -0.3 & 10.2 & -5.8 & -0.1 & -3.5 & -2.1 \\
\hline Jul-99 & 1.8 & 4.2 & -2.0 & 1.1 & 14.0 & -8.1 & -0.2 & -6.2 & -4.0 \\
\hline Aug-99 & 2.9 & 4.2 & -3.0 & 1.2 & 14.7 & -7.2 & 10.0 & -6.2 & -1.0 \\
\hline Sep-99 & 0.7 & 1.4 & -1.5 & 0.8 & 15.6 & -12.5 & 6.0 & -7.7 & -1.4 \\
\hline Oct-99 & 0.0 & 0.5 & -2.3 & 0.8 & 13.5 & -12.0 & 4.0 & -7.7 & -3.3 \\
\hline Nov-99 & 0.5 & 1.8 & -4.5 & 0.7 & 13.8 & -11.1 & 2.8 & -7.7 & -3.6 \\
\hline Dec-99 & -0.5 & -0.2 & -2.5 & 0.1 & 14.4 & -12.1 & 3.2 & -6.3 & -1.7 \\
\hline Jan-00 & 0.4 & -0.3 & -3.4 & 3.1 & 4.4 & -6.3 & 4.4 & -6.1 & -2.0 \\
\hline Feb-00 & -0.3 & -2.5 & -4.1 & 3.1 & 5.3 & -5.1 & 7.8 & -6.1 & -1.9 \\
\hline Mar-00 & 0.2 & -2.0 & -3.2 & 3.1 & 7.6 & -5.5 & 10.3 & -8.1 & -2.4 \\
\hline Apr-00 & -0.9 & -2.8 & 0.3 & 2.6 & 7.0 & -5.9 & 3.8 & -7.3 & 0.4 \\
\hline May-00 & -2.7 & -6.1 & 0.6 & 3.1 & 6.4 & -6.1 & 2.3 & -7.3 & -0.6 \\
\hline Jun- 00 & -3.3 & -7.6 & -4.7 & 2.7 & 6.2 & -5.3 & 4.8 & -5.4 & 1.7 \\
\hline
\end{tabular}

Sources: National Institute of Statistics, Ministry of Planning. 
$-11-$

Table 9. Cambodia: Central Government Operations, 1996-2000

\begin{tabular}{|c|c|c|c|c|c|c|c|c|c|c|c|c|}
\hline & \multirow{2}{*}{\multicolumn{2}{|c|}{$\begin{array}{r}1996 \\
\text { Actual }\end{array}$}} & \multirow{2}{*}{\multicolumn{2}{|c|}{$\begin{array}{r}1997 \\
\text { Actual }\end{array}$}} & \multirow{2}{*}{\multicolumn{2}{|c|}{$\begin{array}{c}1998 \\
\text { Actual } \\
\end{array}$}} & \multirow{2}{*}{\multicolumn{4}{|c|}{1999}} & \multirow{2}{*}{\multicolumn{2}{|c|}{$\begin{array}{r}2000 \\
\text { Budget } \\
\end{array}$}} \\
\hline & & & & & & & & & & & & \\
\hline & $\begin{array}{r}\text { Billions } \\
\text { of riels } \\
\end{array}$ & $\begin{array}{l}\text { Percent } \\
\text { of GDP }\end{array}$ & $\begin{array}{c}\text { Billions } \\
\text { of riels }\end{array}$ & $\begin{array}{l}\text { Percent } \\
\text { of GDP }\end{array}$ & $\begin{array}{l}\text { Biflions } \\
\text { of riels }\end{array}$ & $\begin{array}{l}\text { Percent } \\
\text { of GDP }\end{array}$ & $\begin{array}{r}\text { Bitlions } \\
\text { of riels }\end{array}$ & $\begin{array}{l}\text { Percent } \\
\text { of GDP }\end{array}$ & $\begin{array}{c}\text { Billions } \\
\text { of riels }\end{array}$ & $\begin{array}{l}\text { Percent } \\
\text { of GDP }\end{array}$ & $\begin{array}{l}\text { Billions } \\
\text { of riels }\end{array}$ & $\begin{array}{l}\text { Percent } \\
\text { of GDP }\end{array}$ \\
\hline Total revenue & 749 & 9.0 & 881 & 9.6 & 943 & 9.0 & 1,245 & 10.9 & 1,316 & 11.5 & 1,475 & 11.8 \\
\hline 1. Tax revenue & 534 & 6.4 & 597 & $6.5^{\circ}$ & 679 & 6.5 & 853 & 7.4 & 948 & 8.3 & 1,035 & 8.3 \\
\hline Direct taxes & 27 & 0.3 & 44 & 0.5 & 56 & 0.5 & 81 & 0.7 & 83 & 0.7 & 104 & 0.8 \\
\hline Indirect taxes & 164 & 2.0 & 206 & 2.3 & 248 & 2.4 & 332 & 2.9 & 432 & 3.8 & 439 & 3.5 \\
\hline o.w. Excise taxes (incl on imports) & 57 & 0.7 & 74 & 0.8 & 76 & 0.7 & 80 & 0.7 & 92 & 0.8 & 94 & 0.8 \\
\hline Domestic & 7 & 0.1 & 11 & 0.1 & 16 & 0.2 & 20 & 0.2 & 16 & 0.1 & 22 & 0.2 \\
\hline Import & 50 & 0.6 & 63 & 0.7 & 60 & 0.6 & 60 & 0.5 & 76 & 0.7 & 72 & 0.6 \\
\hline VAT (incl. on imports) ]/ & $\ldots$ & $\cdots$ & $\ldots$ & $\ldots$ & $\ldots$ & $\ldots$ & 231 & 2.0 & 315 & 2.7 & 329 & 2.6 \\
\hline Domestic & $\ldots$ & $\ldots$ & $\ldots$ & $\ldots$ & $\ldots$ & $\ldots$ & 63 & 0.5 & 43 & 0.4 & 73 & 0.6 \\
\hline Inport & $\ldots$ & $\ldots$ & $\ldots$ & $\ldots$ & $\ldots$ & $\cdots$ & 168 & 1.5 & 286 & 2.5 & 286 & 2.3 \\
\hline Refund (-) & $\ldots$ & $\cdots$ & $\cdots$ & $\cdots$ & $\ldots$ & $\ldots$ & 0 & 0.0 & 14. & 0.1 & 30 & 0.2 \\
\hline Trade taxes & 344 & 4.1 & 347 & 3.8 & 376 & 3.6 & 441 & 3.8 & 433 & 3.8 & 492 & 3.9 \\
\hline 2. Nontax revenue & 176 & 2.1 & 271 & 3.0 & 230 & 2.2 & 367 & 3.2 & 355 & 3.1 & 420 & 3.4 \\
\hline o.w. Timber Royalties & 28 & 0.3 & 37 & 0.4 & 23 & 0.2 & 73 & 0.6 & 36 & 0.3 & 52 & 0.4 \\
\hline Enterprises and immobile leases & 19 & 0.2 & 21 & 0.2 & 27 & 0.3 & 31 & 0.3 & 20 & 0.2 & 39 & 0.3 \\
\hline Civil aviation & 17 & 0.2 & 15 & 0.2 & 18 & 0.2 & 27 & 0.2 & 19 & 0.2 & 43 & 0.3 \\
\hline PTT & 64 & 0.8 & 83 & 0.9 & 87 & 0.8 & 161 & 1.4 & 109 & 0.9 & 150 & 1.2 \\
\hline Quota Auction & $\cdots$ & $\cdots$ & $\cdots$ & $\cdots$ & $\cdots$ & $\ldots$ & 0 & 0.0 & 87 & 0.8 & 10 & 0.1 \\
\hline Garment licenses & $\cdots$ & $\cdots$ & $\cdots$ & $\cdots$ & $\cdots$ & $\cdots$ & 0 & 0.0 & 21 & 0.2 & 36 & 0.3 \\
\hline 3. Capital revenue & 39 & 0.5 & 12 & 0.1 & 33 & 0.3 & 25 & 0.2 & 14 & 0.1 & 20 & 0.2 \\
\hline Total expenditure $2 /$ & $144]$ & 17.3 & 1,260 & 13.8 & 1.571 & 14.9 & 1,480 & 12.9 & 1,825 & 15.9 & 2,242 & 18.0 \\
\hline 1. Current expenditure & $81 \hat{3}$ & 9.8 & 808 & 8.8 & 941 & 8.9 & 1,100 & 9.6 & 1,097 & 9.6 & 1,285 & 10.3 \\
\hline Wages & 354 & 4.3 & 382 & 4.2 & 451 & 4.3 & 482 & 4.2 & 525 & 4.6 & $\$ 27$ & 4.2 \\
\hline Civil administration & 125 & 1.5 & 132 & 1.4 & 154 & 1.5 & 167 & 1.5 & 194 & 1.7 & 218 & 1.7 \\
\hline Defense and security & 229 & 2.8 & 250 & 2.7 & 297 & 2.8 & 315 & 2.7 & 331 & 2.9 & 309 & 2.5 \\
\hline Non-wage & 459 & 5.5 & 426 & 4.7 & 490 & 4.7 & 618.2 & 5.4 & 572 & 5.0 & 758 & 6.1 \\
\hline Operating expenditures & 345 & 4.1 & 322 & 3.5 & 372 & 3.5 & 396 & 3.5 & 410 & 3.6 & 505 & 4.0 \\
\hline Civil administration & 166 & 2.0 & 264 & 1.8 & 220 & 2.1 & 265 & 2.3 & 276 & 2.4 & 376 & 3.0 \\
\hline Defense and security & 179 & 2.2 & 158 & 1.7 & 152 & 1.4 & 131 & 1.1 & 134 & 1.2 & 129 & 1.0 \\
\hline Economic transfers & 45 & 0.2 & 7 & 0.1 & 14 & 0.1 & 44 & 0.4 & 12 & 0.1 & 46 & 0.4 \\
\hline Social transfers & 62 & 0.7 & 68 & 0.7 & 71 & 0.7 & 94 & 0.8 & 104 & 0.9 & 117 & 0.9 \\
\hline Civil administration & 55 & 0.7 & 61 & 0.7 & 63 & 0.6 & 85 & 0.7 & 95 & 0.8 & 100 & 0.8 \\
\hline Defense and security & 7 & 0.1 & 7 & 0.1 & 8 & 0.1 & 9 & 0.1 & 9 & 0.1 & 17 & 0.1 \\
\hline Interest & 13 & 0.2 & 10 & 0.1 & 15 & 0.1 & 13 & 0.1 & 22 & 0.2 & 25 & 0.2 \\
\hline Other non-wage & 23 & 0.3 & 18 & 0.2 & 18 & 0.2 & 22 & 0.2 & 21 & 0.2 & 25 & 0.2 \\
\hline Reserve funds & 0 & 0.0 & 2 & 0.0 & 0 & 0.0 & 44 & 0.4 & 0 & 0.0 & 36 & 0.3 \\
\hline Election & 0 & 0.0 & 0 & 0.0 & 0 & 0.0 & 6 & 0.1 & 2 & 0.0 & 5 & 0.0 \\
\hline 2. Capital expenditure & 629 & 7.6 & 452 & 4.9 & 630 & 6.0 & 380 & 3.3 & 728 & 6.3 & 957 & 7.7 \\
\hline Locally firanced & 62 & 0.7 & 110 & 1.2 & 120 & 1.1 & 180 & 1.6 & 224 & 1.9 & 257 & 2.1 \\
\hline Externally financed & 567 & 6.8 & 342 & 3.7 & 510 & 4.8 & 200 & 1.7 & 504 & 4.4 & 700 & 5.6 \\
\hline CURRENT BALANCE & -103 & -1.2 & 61 & 0.7 & -32 & -0.3 & 120 & 1.0 & 205 & 1.8 & 170 & 1.4 \\
\hline OVERALL BAI ANCE & -692 & -8.3 & -379 & -4.1 & -628 & -6.0 & +235 & -2.0 & -509 & -4.4 & -767 & -6.1 \\
\hline OVERALL BALANCE(incl. grants) & .113 & -1.4 & -38 & -0.4 & -286 & -2.7 & -185 & -1.6 & -167 & -1.5 & -217 & -1.7 \\
\hline FINANCING & 692 & 8.3 & 379 & 4.1 & 628 & 6.0 & 235 & 20 & 509 & 4.4 & 767 & 6.1 \\
\hline 1. Foreign financing (net) & 674 & 8.1 & 446 & 4.9 & 505 & 4.8 & 248 & 2.2 & 515 & 4.5 & 843 & 6.8 \\
\hline o.w. Project aid & 566 & 6.8 & 351 & 3.8 & 504 & 4.8 & 200 & 1.7 & 511 & 4.5 & 700 & $\$ .6$ \\
\hline Budget support (grant) & 150 & 3.8 & 96 & 1.0 & 2 & 0.0 & 50 & 0.4 & 4 & 0.0 & 150 & 1.2 \\
\hline 2. Domestic financing & -9 & -0.1 & -57 & -0.6 & 117 & 1.1 & -13 & -0.1 & -45 & -0.4 & .76 & -0.6 \\
\hline Central bank financing & -17 & -0.2 & -75 & -0.8 & 120 & 1.1 & -13 & 0.0 & -70 & -0.6 & .76 & -0.6 \\
\hline Non bank financing & 8 & 0.1 & 18 & 0.2 & -4 & 0.0 & 0 & 0.0 & 26 & 0.2 & 0 & 0.0 \\
\hline 3. Outstanding operations $3 /$ & 28 & 0.3 & -10 & -0.1 & 6 & 0.1 & 0 & 0.0 & 38 & 0.3 & 0 & 0.0 \\
\hline Memorandum items: & & & & & & & & & & & & \\
\hline Defense and security outlays & 415 & 5.0 & 415 & 4.5 & 457 & 4.3 & 455 & 4.0 & 477 & 4.2 & 455 & 3.6 \\
\hline Revenue from forestry & 33 & 0.4 & 41 & 0.4 & 25 & 0.2 & 78 & 0.7 & 49 & 0.4 & $\$ 2$ & 0.4 \\
\hline Total revenue (excl. gament quotas) & 749 & 9.0 & 881 & 9.6 & 943 & 9.0 & 1,245 & 10.9 & 1,229 & 10.7 & 1,465 & 11.7 \\
\hline Health, Education, Rural dev. (commitment) & 129 & 1.6 & 129 & 1.4 & 149 & 1.4 & 208 & 1.8 & 232 & 2.0 & 312 & 2.5 \\
\hline Customs department revenue & 464 & & 486 & & 527 & 5.0 & 668 & 5.8 & 796 & 6.9 & 850 & 6.8 \\
\hline
\end{tabular}

Source: Data provided by Cambodian authorities, and Fund staff estimates.

1/ VAT refunds are adjusted.

2/ Total expenditure is based on a mixture of cash and accrual data.

3/ Includes expenditure committed but not yet allocated to the accounts of the government agencies that execute the budget. 
Table 10. Cambodia: Structure of Revenue, 1996-2000

(In billions of riels)

\begin{tabular}{|c|c|c|c|c|c|}
\hline & 1996 & 1997 & 1998 & 1999 & $\begin{array}{r}2000 \\
\text { Budget }\end{array}$ \\
\hline Total revenue & 749.1 & 881.0 & 942.7 & $1,316.3$ & $1,475.0$ \\
\hline Tax revenue & 534.3 & 597.4 & 679.4 & 947.7 & $1,035.0$ \\
\hline Direct taxes & 26.5 & 43.6 & 55.5 & 82.7 & 103.6 \\
\hline Wage tax & 2.7 & 5.7 & 8.5 & 10.6 & 15.0 \\
\hline Profit tax & 18.5 & 35.0 & 42.1 & 63.8 & 81.6 \\
\hline Public enterprises & 6.0 & 6.4 & 8.6 & 7.0 & 8.0 \\
\hline Private enterprises & 12.5 & 28.6 & 33.5 & 54.2 & 73.6 \\
\hline Petroleum enterprises & $\ldots$ & $\ldots$ & $\ldots$ & 2.6 & 0.0 \\
\hline Property tax & 2.1 & 2.6 & 4.7 & 5.7 & 7.0 \\
\hline Tax on unutilized land & 0.0 & 0.3 & 0.2 & 0.0 & 0.0 \\
\hline Other income taxes & 3.2 & 0.0 & 0.0 & 2.6 & 0.0 \\
\hline Indirect taxes & 163.7 & 206.5 & 247.5 & 431.6 & 439.4 \\
\hline Turnover tax & 27.4 & 46.6 & 65.9 & 21.8 & 12.0 \\
\hline Public entcrprises & 4.2 & 3.0 & 3.9 & 1.3 & 0.0 \\
\hline Private enterprises & 23.2 & 43.6 & 62.1 & 20.5 & 12.0 \\
\hline Consumption tax & 70.4 & 75,2 & 90.1 & $\ldots$ & $\ldots$ \\
\hline VAT & $\ldots$ & $\ldots$ & $\ldots$ & 314.9 & 329.0 \\
\hline Domestic & $\ldots$ & $\ldots$ & $\ldots$ & 42.8 & 73.0 \\
\hline Public enterprises & $\ldots$ & $\ldots$ & $\ldots$ & 2.0 & 3.0 \\
\hline Private enterprises & $\ldots$ & $\ldots$ & $\ldots$ & 39.4 & 70.0 \\
\hline Petroleum companies & $\ldots$ & $\ldots$ & $\ldots$ & 1.3 & 0.0 \\
\hline Import & $\ldots$ & $\ldots$ & $\ldots$ & 285.8 & 286.0 \\
\hline Petroleurn companies & $\ldots$ & $\ldots$ & $\ldots$ & 76.6 & 56.0 \\
\hline Other companies & $\ldots$ & $\ldots$ & $\ldots$ & 209.2 & 230.0 \\
\hline VAT refunds (-) & $\ldots$ & $\ldots$ & $\ldots$ & 13.7 & 30.0 \\
\hline Excise taxes & 56.6 & 74.1 & 76.1 & 91.8 & 94.0 \\
\hline Domestic & 6.8 & 11.1 & 15.9 & 15.5 & 22.0 \\
\hline Import & 49.8 & 63.0 & 60.2 & 76.3 & 72.0 \\
\hline Others & 9.2 & 10.6 & 15.4 & 2.9 & 4.4 \\
\hline Business license tax & 1.6 & 1.9 & 2.1 & 0.1 & 0.0 \\
\hline Vehicle license tax & 3.3 & 3.8 & 7.1 & 0.0 & 0.0 \\
\hline Hotel tax & 0.0 & 0.0 & 0.0 & 0.0 & 0.0 \\
\hline Property transfer tax & 2.4 & 2.5 & 3.5 & 0.0 & 0.0 \\
\hline Stamp tax & 0.7 & 1.0 & 1.1 & 1.3 & 1.9 \\
\hline Penalties & 0.5 & 1.0 & 1.2 & 1.5 & 2.5 \\
\hline Tax on illegally imported cars & 0.4 & 0.1 & 0.0 & 0.0 & 0.0 \\
\hline Other indireet taxes & 0.4 & 0.3 & 0.3 & 0.0 & 0.0 \\
\hline Taxes on international trade & 344.2 & 347.3 & 376.3 & 433.4 & 492.0 \\
\hline Taxes and duties on imports & 334.8 & 335.5 & 372.5 & 415.3 & 480.5 \\
\hline Import tariffs on petroleum & 99.7 & 117.8 & 143.6 & 150.7 & 146.0 \\
\hline Import tariffs (non-petroleum) & 231.5 & 209.8 & 217.5 & 246.4 & 290.0 \\
\hline Fees on petroleum products & 1.8 & 1.8 & 2.0 & 1.9 & 2.5 \\
\hline Import fees & 1.8 & 6.1 & 9.4 & 16.3 & 7.0 \\
\hline Sur tax on petroleum & 0.0 & 0.0 & 0.0 & 0.0 & 35.0 \\
\hline Taxes on exports & 8.2 & 9.9 & 2.7 & 16.5 & 8.5 \\
\hline Timber & 5.6 & 3.3 & 1.7 & 12.7 & 5.0 \\
\hline Rubber & 1.6 & 5.6 & 0.0 & 2.6 & 2.0 \\
\hline Other products & 0.9 & 1.0 & 1.0 & 1.3 & 1.5 \\
\hline Penalties & 1.2 & 2.0 & 1.1 & 1.6 & 3.0 \\
\hline
\end{tabular}

Source: The Ministry of Economy and Finance. 
Table 10. Cambodia: Structure of Revenue, 1996-2000 (concluded)

(In billions of riels)

\begin{tabular}{|c|c|c|c|c|c|}
\hline & 1996 & 1997 & 1998 & 1999 & $\begin{array}{r}2000 \\
\text { Budget }\end{array}$ \\
\hline Nontax revenue & 175.5 & 271.3 & 230.1 & 354.8 & 420.0 \\
\hline Receipts on public property & 92.7 & 134.1 & 120.4 & 89.4 & 171.8 \\
\hline Fisheries & 7.1 & 7.4 & 8.2 & 9.2 & 12.7 \\
\hline Forests & 27.5 & 37.4 & 22.8 & 36.3 & 51.5 \\
\hline Receipts from public enterprises & 34.9 & 36.4 & 55.5 & 30.5 & 65.6 \\
\hline Factory leases & 10.1 & 8.4 & 8.7 & 8.8 & 16.5 \\
\hline Amortization payments & 1.0 & 4.0 & 5.6 & 1.1 & 1.9 \\
\hline Surplus on working capital & 0.6 & 0.1 & 0.0 & 0.0 & 0.0 \\
\hline Operating surpluses of public enterprises & 0.0 & 0.0 & 9.2 & 0.0 & 0.0 \\
\hline Civil aviation & 17.1 & 17.1 & 17.7 & 19.2 & 42.6 \\
\hline Tourism receipts & 0.0 & 1.0 & 0.7 & 0.8 & 4.2 \\
\hline Sale of material & 0.0 & 0.3 & 0.4 & 0.0 & 0.0 \\
\hline Transportation fees & 0.3 & 0.2 & 0.2 & 0.0 & 0.1 \\
\hline Receipts from commercial activity & 0.2 & 0.0 & 0.0 & 0.0 & 0.0 \\
\hline Sales of electricity to provinces & 0.0 & 0.1 & 0.0 & 0.0 & 0.0 \\
\hline Receipts from water supply & 4.1 & 0.8 & 0.0 & 0.0 & 0.0 \\
\hline Others & 1.4 & 4.4 & 13.0 & 0.4 & 0.4 \\
\hline Royalties and concessions & 6.2 & 33.2 & 3.5 & 1.8 & 18.9 \\
\hline Petroleum concessions & 0.9 & 31.6 & 2.5 & 0.7 & 0.4 \\
\hline Mining concessions & 0.2 & 0.5 & 0.3 & 0.2 & 2.0 \\
\hline Others & 5.1 & 1.1 & 0.7 & 0.9 & 16.5 \\
\hline User fees & 8.0 & 6.6 & 10.9 & 0.1 & 0.0 \\
\hline Lease of market stands & 4.7 & 4.1 & 8.0 & 0.0 & 0.0 \\
\hline Pre-paid fees for car parking & 1.3 & 0.8 & 1.0 & 0.1 & 0.0 \\
\hline Pre-paid fees for ferries & 1.5 & 1.2 & 1.3 & 0.0 & 0.0 \\
\hline Pre-paid fees for auction stands & 0.4 & 0.4 & 0.5 & 0.0 & 0.0 \\
\hline Building leases & 8.7 & 12.6 & 18.8 & 11.5 & 23.0 \\
\hline Other & 0.2 & 0.6 & 0.8 & 0.0 & 0.2 \\
\hline Other nontax revenue & 82.9 & 137.2 & 109.7 & 265.5 & 248.2 \\
\hline Posts and telecommunication & 63.6 & 83.0 & 87.2 & 108.9 & 150.3 \\
\hline Ratio-TV (advertisement) & 0.1 & 0.0 & 0.0 & 0.0 & 0.0 \\
\hline Public services & 12.8 & 46.0 & 17.5 & 141.9 & 79.0 \\
\hline o.w. Visa fees & 8.0 & 23.8 & 7.2 & 17.2 & 23.6 \\
\hline Timber export license fee & 0.0 & 0.0 & 0.0 & 0.0 & 0.0 \\
\hline Product quality control & 0.7 & 1.7 & 5.3 & 7.8 & 4.8 \\
\hline License fee for establishing companies & 0.3 & 0.2 & 0.3 & 1.4 & 0.8 \\
\hline Car sticker & 1.2 & 1.3 & 1.2 & 1.5 & 2.0 \\
\hline Other documents & 0.0 & 2.8 & 1.9 & 0.6 & 0.4 \\
\hline Sales of tax documents & 0.0 & 0.1 & 0.1 & 0.1 & 0.1 \\
\hline Sales of number plates & 0.4 & 0.5 & 0.7 & 0.5 & 0.6 \\
\hline Quota auction and export licenses & $\ldots$ & $\ldots$ & $\ldots$ & 108.1 & 46.0 \\
\hline Others & 2.2 & 0.8 & 0.8 & 4.7 & 0.7 \\
\hline Interest & 2.9 & 0.0 & 3.4 & 0.0 & 0.0 \\
\hline Nontax revenue of provinces & 1.4 & 0.1 & 0.1 & 0.1 & 0.0 \\
\hline Banking profit & $\ldots$ & $\ldots$ & $\ldots$ & 10.0 & 15.0 \\
\hline Other nontax revenue & 2.2 & 8.1 & 1.6 & 4.6 & 3.9 \\
\hline Capital revenue & 39.2 & 12.3 & 33.2 & 13.7 & 20.0 \\
\hline Sales of immobile property & 1.3 & 0.9 & 0.9 & 0.5 & 0.0 \\
\hline Sales of land & 0.0 & 0.9 & 0.1 & 0.0 & 0.0 \\
\hline Privatization & 21.8 & 10.5 & 32.2 & 13.2 & 20.0 \\
\hline Other & 16.0 & 0.0 & -0.0 & 0.0 & 0.0 \\
\hline
\end{tabular}

Source: The Ministry of Economy and Finance. 
Table 11. Cambodia: Budgetary Expenditure by Economic Classification, 1996-2000 1/

\begin{tabular}{|c|c|c|c|c|c|}
\hline & 1996 & 1997 & 1998 & 1999 & $\begin{array}{r}2000 \\
\text { Budget }\end{array}$ \\
\hline & \multicolumn{4}{|c|}{ (In billions of riel) } & \\
\hline Total expenditure & $1,441.5$ & $1,259.7$ & $1,571.2$ & $1,825.0$ & $2,242.0$ \\
\hline Current expenditure & 812.9 & 807.8 & 941.2 & $.1,097.2$ & $1,285.0$ \\
\hline Salaries & 354.2 & 381.9 & 451.1 & 525.4 & 527.3 \\
\hline Civil administration & 125.3 & 132.2 & 154.3 & 194.1 & 218.1 \\
\hline Defense and security & 228.9 & 249.7 & 296.8 & 331.3 & 309.3 \\
\hline Operating costs & 344.9 & 321.9 & 371.7 & 410.1 & 504.6 \\
\hline Civil administration & 165.7 & 163.7 & 219.6 & 276.0 & 375.6 \\
\hline Defense and security & 179.2 & 158.3 & 152.2 & 134.1 & 129.0 \\
\hline Economic transfers & 15.5 & 6.6 & 14.3 & 12.0 & 45.7 \\
\hline Social transfers & 62.0 & 68.0 & 70.9 & 103.8 & 116.5 \\
\hline Civil administration & 55.1 & 60.8 & 62.8 & 94.6 & 99.8 \\
\hline Defense and security & 6.9 & 7.1 & 8.0 & 9.2 & 16.8 \\
\hline Interest & 12.8 & 9.5 & 14.7 & 22.4 & 25.0 \\
\hline Other current expenditure & 23.4 & 19.9 & 18.4 & 23.5 & 65.9 \\
\hline o.w. Subsidy to provinces & 17.2 & 16.7 & 16.3 & 16.0 & 26.0 \\
\hline Contribution to international org. & 6.2 & 1.1 & 2.1 & 4.9 & 14.9 \\
\hline Capital expenditure & 628.6 & 451.9 & 630.0 & 727.8 & 957.0 \\
\hline Locally financed & 61.6 & 110.3 & 120.4 & 223.6 & 257.0 \\
\hline \multirow[t]{2}{*}{ Externally financed } & 567.0 & 341.6 & 509.5 & 504.2 & 700.0 \\
\hline & \multicolumn{4}{|c|}{ (In percent of total expenditure) } & \\
\hline Total & 100.0 & 100.0 & 100.0 & 100.0 & 100.0 \\
\hline Current expenditure & 56.4 & 64.1 & 59.9 & 60.1 & 57.3 \\
\hline Salaries & 24.6 & 30.3 & 28.7 & 28.8 & 23.5 \\
\hline Civil administration & 8.7 & 10.5 & 9.8 & 10.6 & 9.7 \\
\hline Defense and security & 15.9 & 19.8 & 18.9 & 18.2 & 13.8 \\
\hline Operating costs & 23.9 & 25.6 & 23.7 & 22.5 & 22.5 \\
\hline Civil administration & 11.5 & 13.0 & 14.0 & 15.1 & 16.8 \\
\hline Defense and security & 12.4 & 12.6 & 9.7 & 7.3 & 5.8 \\
\hline Economic transfers & 1.1 & 0.5 & 0.9 & 0.7 & 2.0 \\
\hline Social transfers & 4.3 & 5.4 & 4.5 & 5.7 & 5.2 \\
\hline Civil administration & 3.8 & 4.8 & 4.0 & 5.2 & 4.4 \\
\hline Defense and security & 0.5 & 0.6 & 0.5 & 0.5 & 0.7 \\
\hline Interest & 0.9 & 0.8 & 0.9 & 1.2 & 1.1 \\
\hline Other current expenditure & 1.6 & 1.6 & 1.2 & 1.3 & 2.9 \\
\hline o.w. Subsidy to provinces & 1.2 & 1.3 & 1.0 & 0.9 & 1.2 \\
\hline Contribution to international org. & 0.4 & 0.1 & 0.1 & 0.3 & 0.7 \\
\hline Capital expenditure & 43.6 & 35.9 & 40.1 & 39.9 & 42.7 \\
\hline Locally financed & 4.3 & 8.8 & 7.7 & 12.3 & 11.5 \\
\hline Externally financed & 39.3 & 27.1 & 32.4 & 27.6 & 31.2 \\
\hline
\end{tabular}

Source: The Ministry of Economy and Finance

1/ For 1996-99, cash and accrual basis. 
Table 12. Cambodia: Budgetary Expenditure by Ministry, 1998-2000 1/

\begin{tabular}{|c|c|c|c|c|c|c|c|c|c|c|c|c|c|c|c|c|c|}
\hline \multirow[b]{2}{*}{ Ministry } & & \multicolumn{5}{|c|}{1998} & \multicolumn{5}{|c|}{1999} & \multicolumn{6}{|c|}{2000 (Budget) } \\
\hline & & Total & Salaries & $\begin{array}{l}\text { Operating } \\
\text { Costs }\end{array}$ & Other & Capital $2 /$ & Total & Salaries & $\begin{array}{l}\text { Operating } \\
\text { Costs }\end{array}$ & Other & Capital $2 /$ & Total & Salaries & $\begin{array}{c}\text { Operating } \\
\text { Costs } \\
\end{array}$ & Other & Capital & 21 \\
\hline & & \multicolumn{16}{|c|}{ (In billions of riels) } \\
\hline Total & & 1,054 & 448 & 369 & 121 & 116 & 1,319 & 518 & 404 & 179 & 218 & 1,542 & 527 & 505 & 224 & & 257 \\
\hline Royal Palace & & 13 & 4 & 3 & 7 & 0 & 20 & 6 & 3 & 11 & 0 & 19 & 6 & 4 & 9 & & 0 \\
\hline National Assembly & & 12 & 9 & 3 & 0 & 0 & 13 & 8 & 5 & 0 & 0 & 18 & 10 & 6 & 2 & & 0 \\
\hline Senate & & $\ldots$ & $\ldots$ & $\cdots$ & $\ldots$ & $\ldots$ & 6 & 3 & 3 & 0 & 0 & 9 & 5 & 4 & 0 & & 0 \\
\hline Constitutional Council & & $\ldots$ & $\ldots$ & $\ldots$ & $\ldots$ & $\ldots$ & 2 & 1 & 1 & 0 & 0 & 2 & 1 & 1 & 0 & & 0 \\
\hline Council of Ministers & & 57 & 7 & 40 & 7 & 3 & 54 & 4 & 33 & 12 & 6 & 40 & 4 & 32 & 5 & & 0 \\
\hline Foreign Affairs & & 26 & 11 & 13 & 2 & 0 & 37 & 13 & 22 & 2 & 1 & 50 & 13 & 25 & 12 & & 0 \\
\hline National Defense & & 312 & 213 & 93 & 7 & 0 & 336 & 236 & 92 & 8 & 0 & 311 & 206 & 90 & 15 & & 0 \\
\hline Interior & & 173 & 87 & 66 & 21 & 0 & 147 & 91 & 46 & 7 & 2 & 153 & 103 & 46 & 3 & & 0 \\
\hline National Assembly Relation and Inspection & & $\ldots$ & $\ldots$ & $\ldots$ & $\ldots$ & $\ldots$ & 0 & 0 & 0 & 0 & 0 & 1 & 0 & 1 & 0 & & 0 \\
\hline Economy and Finance & & 109 & 3 & 18 & 27 & 62 & 134 & 3 & 22 & 69 & 40 & 31 & 3 & 27 & 1 & & 0 \\
\hline Information & & 16 & 1 & 3 & 0 & 11 & 8 & 1 & 3 & 0 & 4 & 6 & 2 & 4 & 0 & & 0 \\
\hline Public Works and Transport & & 13 & 4 & 1 & 1 & 7 & 29 & 4 & 3 & 2 & 20 & 47 & 4 & 5 & 38 & & $\theta$ \\
\hline Agriculture, Forestry and Fishery & & 18 & 7 & 8 & 1 & 3 & 21 & 7 & 11 & 3 & 0 & 26 & 8 & 13 & 5 & & 0 \\
\hline Justice & & 2 & 1 & 1 & 0 & 0 & 5 & 1 & 3 & 0 & 0 & 7 & 0 & 6 & 1 & & 0 \\
\hline Education, Youth and Sport & & 102 & 80 & 20 & 2 & 0 & 156 & 112 & 35 & 3 & 6 & 183 & 126 & 50 & 7 & & 0 \\
\hline Commerce & & 2 & 1 & 1 & 0 & 0 & 4 & 1 & 3 & 0 & 0 & 7 & 1 & 4 & 1 & & 0 \\
\hline Industry, Mine and Energy & & 3 & 1 & 1 & 1 & 0 & 4 & 1 & 2 & 1 & 0 & 6 & 2 & 3 & 1 & & 0 \\
\hline Planning & & 2 & 1 & 1 & 0 & 0 & 2 & 1 & 1 & 0 & 0 & 3 & 1 & 2 & 0 & & 0 \\
\hline Health & & 44 & 11 & 33 & 1 & 0 & 126 & 12 & 62 & 1 & 50 & 121 & 16 & 104 & 2 & & 0 \\
\hline Tourism & & 2 & 1 & 1 & 0 & 0 & 5 & 1 & 4 & 0 & 0 & 7 & 1 & 6 & 0 & & 0 \\
\hline Public Worship and Religion & & 1 & 1 & 0 & 0 & 0 & 1 & 1 & 1 & 0 & 0 & 2 & 1 & 1 & 0 & & 0 \\
\hline Posts and Telecommunication & & 54 & 2 & 28 & 0 & 24 & 114 & 2 & 36 & 0 & 76 & 42 & 2 & 39 & 0 & & 0 \\
\hline Culture and Fine Arts & & 3 & 2 & 1 & 0 & 0 & 5 & 3 & 2 & 0 & 0 & 8 & 3 & 4 & 0 & & 0 \\
\hline Social Affairs, Labor and Vocational Training & & 48 & 1 & 1 & 45 & 1 & 19 & I & 2 & 16 & 0 & 27 & 1 & 4 & 22 & & 0 \\
\hline Environment & & 1 & 1 & 1 & 0 & 0 & 2 & 1 & 1 & 0 & 0 & 4 & 1 & 3 & 0 & & 0 \\
\hline Rural Development & & 6 & 1 & 1 & 0 & 4 & 16 & 1 & 2 & 0 & 12 & 8 & 1 & 6 & 0 & & 0 \\
\hline Woman Affair and Veteran & & 1 & 0 & 0 & 0 & 0 & 44 & 1 & 1 & 41 & 1 & 58 & 1 & 4 & 53 & & 0 \\
\hline Urbanization and Constitution & & 1 & 0 & 0 & 0 & 0 & 2 & 1 & 1 & 0 & 0 & 4 & 1 & 3 & 0 & & 0 \\
\hline Water Resources and Metrology & & $\ldots$ & $\ldots$ & $\ldots$ & $\ldots$ & $\ldots$ & 3 & 0 & 3 & 0 & 0 & 7 & 1 & 6 & 0 & & 0 \\
\hline National Election Committee & & 32 & 0 & 32 & 0 & 0 & $\ldots$ & $\ldots$ & $\ldots$ & $\ldots$ & $\ldots$ & 3 & 1 & 2 & 0 & & 0 \\
\hline \multirow[t]{2}{*}{ Other } & & 0 & 0 & 0 & 0 & 0 & 2 & 0 & 0 & 2 & 0 & 333 & 0 & 0 & 46 & & 257 \\
\hline & \multicolumn{17}{|c|}{ (In percent of total budgetary expenditure) } \\
\hline \multicolumn{18}{|l|}{ Memorandum items: } \\
\hline National Defense & & 30 & 48 & 25 & 6 & 0 & 25 & 46 & 23 & 5 & 0 & 20 & 39 & 18 & 7 & & 0 \\
\hline Interior & & 16 & 19 & 18 & 17 & 0 & 11 & 18 & 11 & 4 & 1 & 10 & 20 & 9 & 1 & & 0 \\
\hline Economy and Finance & & 10 & 1 & $s$ & 22 & 54 & 10 & 1 & 6 & 38 & 18 & 2 & 1 & 5 & 0 & & 0 \\
\hline Public Works & & 1 & 1 & 0 & 1 & 6 & 2 & 1 & 1 & 1 & 9 & 3 & 1 & 1 & 17 & & 0 \\
\hline Agriculture & & 2 & 1 & 2 & 1 & 3 & 2 & 1 & 3 & 2 & 0 & 2 & 2 & 3 & 2 & & 0 \\
\hline Education & & 10 & 18 & 5 & 2 & 0 & 12 & 22 & 9 & 2 & 3 & 12 & 24 & 10 & 3 & & 0 \\
\hline Industry & 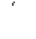 & 0 & 0 & 0 & 1 & 0 & 0 & 0 & 0 & 1 & 0 & 0 & 0 & 1 & 1 & & 0 \\
\hline Health & & 4 & 2 & 9 & 0 & 0 & 10 & 2 & 15 & 0 & 23 & 8 & 3 & 21 & 1 & & 0 \\
\hline
\end{tabular}

Source: The Ministry of Economy and Finance.

$1 /$ Commitment basis.

2/ Excludes externally financed capital expenditure. 
Table 13. Cambodia: Official External Assistance to the Budget, 1996-2000 (In billions of riels)

\begin{tabular}{|c|c|c|c|c|c|}
\hline & 1996 & 1997 & 1998 & 1999 & $\begin{array}{r}2000 \\
\text { Budget }\end{array}$ \\
\hline Total official external financing & 674.2 & 445.9 & 505.4 & 515.4 & 843.4 \\
\hline Budget support & 149.9 & 96.0 & 1.5 & 4.4 & 150.0 \\
\hline Grants & 75.8 & 46.2 & 1.5 & 4.4 & 0.0 \\
\hline Loans & 74.0 & 49.8 & 0.0 & 0.0 & 150.0 \\
\hline World Bank & 74.0 & 7.2 & 0.0 & 0.0 & $\ldots$ \\
\hline AsDB & 0.0 & 42.5 & 0.0 & 0.0 & $\ldots$ \\
\hline Project aid & 565.8 & 350.8 & 503.9 & 511.0 & 700.0 \\
\hline Grants & 429.8 & 244.6 & 340.7 & 337.4 & 400.0 \\
\hline Loans & 137.2 & 97.0 & 168.9 & 166.8 & 300.0 \\
\hline World Bank & 34.1 & 66.4 & 56.3 & 69.8 & 100.0 \\
\hline AsDB & 103.1 & 30.6 & 112.6 & 96.9 & 200.0 \\
\hline Pending & -1.2 & 9.2 & -5.7 & 6.8 & 0.0 \\
\hline Amortization & -41.4 & -0.9 & 0.0 & 0.0 & -6.6 \\
\hline
\end{tabular}

Source: The Ministry of Economy and Finance. 
Table 14. Cambodia: Profile of the Commercial Bank System

(As of July 2000)

\begin{tabular}{|c|c|c|c|c|}
\hline Name of Banks & $\begin{array}{c}\text { No. of } \\
\text { Branches }\end{array}$ & $\begin{array}{r}\text { Authorized } \\
\text { Capital (000 U.S.\$) }\end{array}$ & $\begin{array}{c}\text { Date of } \\
\text { Operation }\end{array}$ & $\begin{array}{c}\text { Majority } \\
\text { Shareholder }\end{array}$ \\
\hline \multicolumn{5}{|l|}{ State-owned banks (2) } \\
\hline Foreign Trade Bank & & 385 million riels & Oct. 10,1979 & \\
\hline Rural Development Bank & & 10 billion riels & Jun. 22,1998 & \\
\hline \multicolumn{5}{|l|}{ Foreign bank branches (7) } \\
\hline Krung Thai Bank Public Co. Ltd. & & 5,000 & Sep. 25,1992 & Thai \\
\hline Bangkok Bank Public Co. Ltd. & & 5,000 & Sep. 26,1992 & Thai \\
\hline Thai Farmers Bank Public Co. Ltd. & & 5,000 & Aug. 21, 1992 & Thai \\
\hline Credit Agricole Indosuez & & 5,000 & Mar. 1, 1993 & French \\
\hline May Bank & & 5,000 & Dec. 28,1993 & Malaysian \\
\hline First Commercial Bank & & 13,000 & Sep. 23,1998 & Taiwan POC \\
\hline Standard Charted Bank & & 13,000 & Feb 25. 1999 & British \\
\hline \multicolumn{5}{|l|}{ Private banks (22) } \\
\hline Cambodia Commercial Bank & 3 & 10,000 & Jul. 1, 1991 & Thai \\
\hline Canadia Bank Ltd. & 6 & 5,000 & Nov. 11,1991 & Canadian \\
\hline Agricultural and Commercial Bank of Cambodia & & 5,000 & Jul. 9,1992 & Thai \\
\hline Cambodia Public Bank & & 10,000 & May. 25, 1992 & Malaysian \\
\hline Cambodia Farmers Bank 1/ & 1 & 5,000 & Apr. 5, 1992 & Thai \\
\hline Cambodia Asia Bank & & 10,000 & Feb. 23,1993 & Malaysian \\
\hline Chansavangwonk Ban $1 /$ & & 5,000 & May. 19, 1993 & Thai \\
\hline Phnom Penh City Bank 1/ & & 5,000 & Sep. 15,1993 & Thai \\
\hline Singapore Banking Corp. & & 5,000 & Oct. 27,1993 & Singaporean \\
\hline Singapore Commercial Bank & & 5,000 & Dec. 25,1993 & Singaporean \\
\hline Rich Nation Bank & & 5,000 & Jan. 25,1994 & Hong Kong \\
\hline Union Commercial Bank & & 5,000 & Apr. 20, 1994 & Hong Kong \\
\hline Global Commercial Bank & & 5,000 & Apr. 26,1994 & Thai \\
\hline Pacific Commercial Bank & 9 & 7,000 & May 28,1994 & Cambodian \\
\hline First Oversea Bank & 2 & 5,000 & May 30,1994 & Malaysian \\
\hline Great International Bank & & 5,000 & May 30,1994 & Australian \\
\hline Emperor International Bank & & 10,000 & May 31,1994 & Hong Kong \\
\hline Cambodia Mekong Bank & & 10,000 & Jun. 4,1994 & Cambodian \\
\hline Cambodia International Bank & & 5,000 & Jun. 30, 1994 & Singaporean \\
\hline Cambodia Agricultural Industrial and Merchandise Bar & ank & 10 billion riels & Jun. 29, 1996 & Japanese \\
\hline Advanced Bank of Asia Ltd. & & 7,500 & Oct. 25,1996 & Korean \\
\hline Angkor Bank & & 10 billion riels & Feb. 4, 1998 & Cambodian \\
\hline
\end{tabular}

Source: The National Bank of Cambodia.

1/ Banking licenses are suspended. 
Table 15. Cambodia: Monetary Survey, 1997-2000

(in billions of riels; unless otherwise indicated)

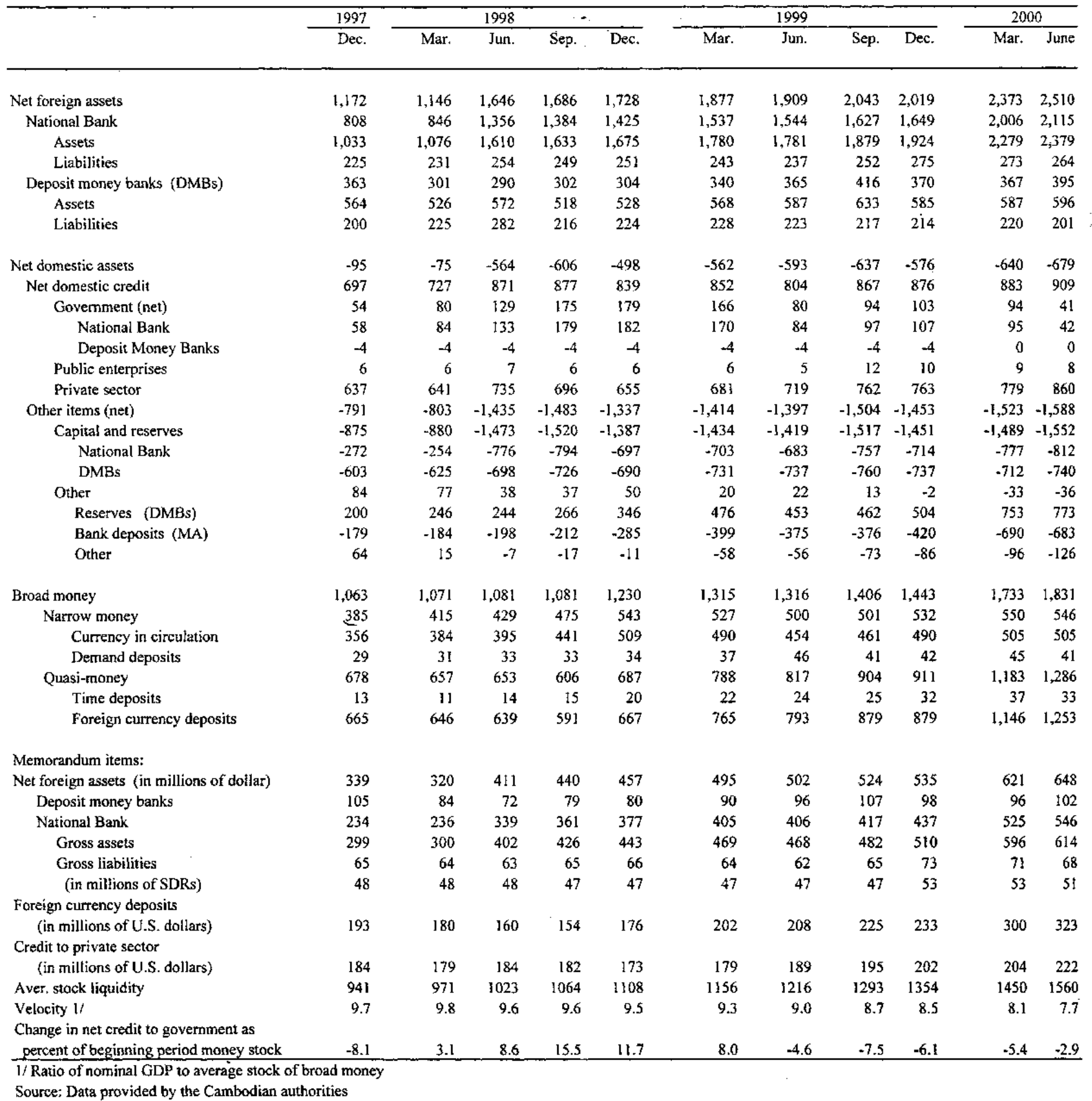


Table 16. Cambodia: Sources of Broad Money Growth, 1997-2000

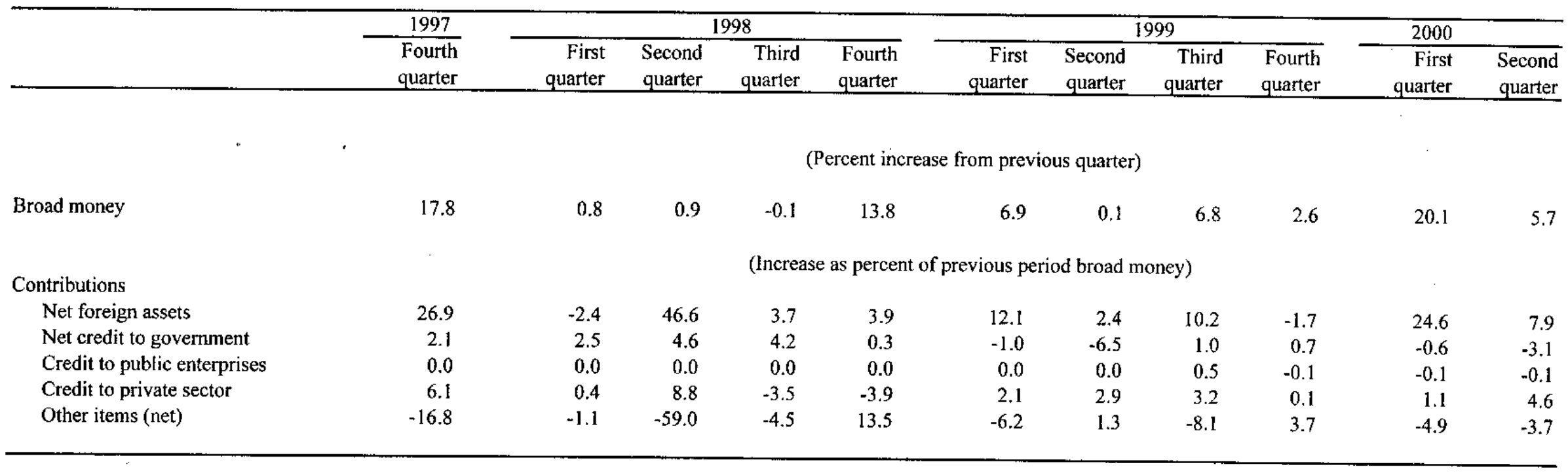

Source: Data provided by the Cambodian authorities 
Table 17. Cambodia: Deposit Money Bank's Balance Sheet, 1997-2000 (In billions of riels)

\begin{tabular}{|c|c|c|c|c|c|c|c|c|c|c|c|}
\hline & \multirow{2}{*}{$\frac{1997}{\text { Dec. }}$} & \multicolumn{4}{|c|}{1998} & \multicolumn{4}{|c|}{1999} & \multicolumn{2}{|c|}{2000} \\
\hline & & Mar. & Jun. & Sep. & Dec. & Mar. & Jun. & Sep. & Dec. & Mar. & Jun. \\
\hline Net foreign assets & 363 & 301 & 290 & 302 & 304 & 340 & 365 & 416 & 370 & 367 & 395 \\
\hline Foreign assets & 564 & 526 & 572 & 518 & 528 & 568 & 587 & 633 & 585 & 587 & .596 \\
\hline Foreign liabilities & 200 & 225 & 282 & 216 & 224 & 228 & 223 & 217 & 214 & 220 & 201 \\
\hline Net domestic assets & 144 & 141 & 153 & 71 & 71 & 9 & 45 & 67 & 79 & 109 & 158 \\
\hline Net claims on government & -4 & -4 & -4 & -4 & -4 & -4 & -4 & -4 & -4 & 0 & 0 \\
\hline Net claims & -4 & -4 & -4 & -4 & -4 & -4 & -4 & -4 & -4 & 0 & 0 \\
\hline Claims & 0 & 0 & 0 & 0 & 0 & 0 & 0 & 0 & 0 & 0 & 0 \\
\hline Deposits & 4 & 4 & 4 & 4 & 4 & 4 & 4 & 4 & 4 & 1 & 1 \\
\hline Claims - treasury bill holdings & 0 & 0 & 0 & 0 & 0 & 0 & 0 & 0 & 0 & 0 & 0 \\
\hline Claims on public enterprises & 6 & 6 & 7 & 6 & 6 & 6 & 5 & 12 & 10 & 9 & 8 \\
\hline Claims on private sector $1 /$ & 637 & 641 & 735 & 696 & 655 & 681 & 719 & 762 & 763 & 779 & 860 \\
\hline Net claims on National Bank & 14 & 9 & 14 & 16 & 14 & 14 & 13 & 11 & 11 & 9 & 5 \\
\hline Claims & 21 & 21 & 23 & 21 & 20 & 23 & 22 & 19 & 19 & 18 & 16 \\
\hline Liabilities & 7 & 12 & 9 & 5 & 6 & 9 & 9 & 8 & 8 & 9 & 10 \\
\hline Other assets (net) & -509 & -511 & -599 & -643 & -599 & -687 & -689 & -713 & -702 & -688 & -714 \\
\hline Assets & 229 & 237 & 253 & 237 & 248 & 237 & 238 & 254 & 251 & 277 & 281 \\
\hline Fixed assets & 110 & 109 & 122 & 124 & 120 & 114 & 112 & 112 & 109 & 110 & 108 \\
\hline Interbank claims & 32 & 48 & 29 & 29 & 55 & 51 & 40 & 49 & 57 & 74 & 76 \\
\hline Other assets & 88 & 80 & 102 & 83 & 73 & 73 & 85 & 93 & 86 & 93 & 97 \\
\hline Liabilities & 738 & 749 & 852 & 880 & 847 & 924 & 926 & 967 & 953 & 965 & 995 \\
\hline Capital & 603 & 600 & 679 & 713 & 690 & 766 & 779 & 793 & 767 & 755 & 778 \\
\hline Capital and reserves & 603 & 625 & 698 & 726 & 690 & 731 & 737 & 760 & 737 & 712 & 740 \\
\hline Provisions & -12 & -40 & -36 & -30 & -22 & 16 & 24 & 14 & 12 & 24 & 18 \\
\hline Valuation adjustment & 12 & 15 & 18 & 17 & 22 & 19 & 18 & 19 & 19 & 19 & 20 \\
\hline Restricted deposits & 4 & 4 & 5 & 4 & 4 & 4 & 4 & $\dot{4}$ & 4 & 2 & 2 \\
\hline Interbank liabilities & 26 & 39 & 25 & 16 & 29 & 30 & 30 & 43 & 49 & 59 & 72 \\
\hline Other & 105 & 104 & 143 & 147 & 125 & 124 & 113 & 128 & 132 & 148 & 144 \\
\hline Reserves & 200 & 246 & 244 & 266 & 346 & 476 & 453 & 462 & 504 & 753 & 773 \\
\hline Cash & 10 & 14 & 6 & 9 & 8 & 21 & 21 & 20 & 20 & 18 & 28 \\
\hline Deposits at National Bank & 190 & 232 & 237 & 256 & 338 & 455 & 432 & 442 & 484 & 735 & 745 \\
\hline Other credits to National Bank & 0 & 0 & 0 & 0 & 0 & 0 & 0 & 0 & 0 & 0 & 0 \\
\hline Deposits & 707 & 687 & 686 & 639 & 721 & 825 & 862 & 945 & 953 & 1,229 & 1,327 \\
\hline Demand deposits & 29 & 31 & 33 & 33 & 34 & 37 & 46 & 41 & 42 & 45 & 41 \\
\hline Time and savings deposits & 13 & 11 & 14 & 15 & 20 & 22 & 24 & 25 & 32 & 37 & 33 \\
\hline Foreign currency deposits & 665 & 646 & 639 & 591 & 667 & 765 & 793 & 879 & 879 & 1,146 & 1,253 \\
\hline
\end{tabular}

Sources: Data provided by the Cambodian authorities.

1/ Almost all in foreign currency. 
Table 18. Cambodia: Reserve Money, 1997-2000

(In billions of riels, unless otherwise indicated)

\begin{tabular}{|c|c|c|c|c|c|c|c|c|c|c|c|}
\hline & \multirow{2}{*}{$\frac{1997}{\text { Dec. }}$} & \multicolumn{3}{|c|}{1998} & \multirow{2}{*}{$\frac{\ddots}{\text { Dec }}$} & \multicolumn{3}{|c|}{1999} & \multirow[b]{2}{*}{$\overline{\text { Dec. }}$} & \multicolumn{2}{|c|}{2000} \\
\hline & & Mar. & Jun. & Sep. & & $\mathrm{Mar}$ & Jun. & Sep. & & Mar. & Jun. \\
\hline Reserve money & 545 & 582 & 600 & 663 & 803 & 909 & 849 & 856 & 930 & 1212 & 1216 \\
\hline Currency outside banks & 356 & 384 & 395 & 441 & 509 & 490 & 454 & 461 & 490 & 505 & 505 \\
\hline Currency in banks & 10 & 14 & 6 & 9 & 8 & 21 & 21 & 20 & 20 & 18 & 28 \\
\hline Bank deposits & 179 & 184 & 198 & 212 & 285 & 399 & 375 & 376 & 420 & 690 & $683^{\circ}$ \\
\hline Required & 44 & 65 & 71 & 61 & 63 & 67 & 72 & 74 & 83 & 99 & 104 \\
\hline Other & 136 & 119 & 127 & 151 & 222 & 332 & 303 & 302 & 337 & 591 & 579 \\
\hline (acct 063$) 1 /$ & 126 & 116 & 119 & 145 & 215 & 327 & 295 & 292 & 330 & 587 & 574 \\
\hline Other deposits & 0 & 0 & 0 & 0 & 0 & 0 & 0 & 0 & 0 & 0 & 0 \\
\hline (acct 063 ) in millions dollars & 39 & 33 & 32 & 39 & 59 & 87 & 80 & 77 & 89 & 155 & 149 \\
\hline Net foreign assets & 808 & 846 & 1356 & 1384 & 1425 & 1537 & 1544 & 1627 & 1649 & 2006 & 2115 \\
\hline Foreign assets & 1033 & 1076 & 1610 & 1633 & 1675 & 1780 & 1781 & 1879 & 1924 & 2279 & 2379 \\
\hline Gold & 0 & 0 & 469 & 517 & 434 & 423 & 397 & 463 & 439 & 424 & 448 \\
\hline Bank accounts & 658 & 700 & 435 & 415 & 490 & 644 & 370 & 374 & 471 & 539 & 518 \\
\hline Foreign exchange & 25 & 15 & 17 & 39 & 41 & 35 & 18 & 27 & 37 & 54 & 44 \\
\hline SDR holdings & 41 & 41 & 42 & 40 & 37 & 35 & 31 & 32 & 20 & 19 & 7 \\
\hline IMF Reserve Position & 0 & 0 & 0 & 0 & 0 & 0 & 0 & 0 & 0 & 0 & 0 \\
\hline Other $2 /$ & 309 & 320 & 646 & 622 & 673 & 643 & 966 & 982 & 956 & 1243 & 1362 \\
\hline Foreign liabilities & 225 & 231 & 254 & 249 & 251 & 243 & 237 & 252 & 275 & 273 & 264 \\
\hline IMF & 225 & 231 & 254 & 249 & 251 & 243 & 237 & 252 & 275 & 273 & 264 \\
\hline Other & 0 & 0 & 0 & 0 & 0 & 0 & 0 & 0 & 0 & 0 & 0 \\
\hline Net Domestic Assets & -249 & -264 & .756 & .722 & -622 & -628 & -695 & -771 & -719 & .794 & -903 \\
\hline Net Credit to Govemment & 58 & 84 & 133 & 179 & 182 & 170 & 84 & 97 & 107 & 95 & 42 \\
\hline Net claims & 58 & 84 & 133 & 179 & 182 & 170 & 84 & 97 & 107 & 95 & 42 \\
\hline Claims & 211 & 233 & 261 & 289 & 289 & 285 & 284 & 285 & 283 & 278 & 278 \\
\hline Deposits & 153 & 148 & 128 & 110 & 106 & 115 & 200 & 187 & 176 & 183 & 236 \\
\hline Net claims on banks & -51 & -60 & -69 & -66 & -67 & -72 & -72 & -77 & -77 & -78 & .80 \\
\hline Claims on banks & 6 & 10 & 7 & 7 & 8 & 7 & 7 & 7 & 5 & 5 & 5 \\
\hline Restricted deposits & 43 & 56 & 61 & 66 & 69 & 73 & 73 & 78 & 76 & 77 & 79 \\
\hline Loans from DMBs & 14 & 14 & 15 & 6 & 6 & 6 & 6 & 6 & 6 & 6 & 6 \\
\hline Due to FTBC $3 /$ & 0 & 0 & 0 & 0 & 0 & 0 & 0 & 0 & 0 & 0 & 0 \\
\hline Claims on public enterprises & 0 & 0 & 0 & 0 & 0 & 0 & 0 & 0 & 0 & 0 & 0 \\
\hline Claims on private sector & 0 & 0 & 0 & 0 & 0 & 0 & 0 & 0 & 0 & 0 & 0 \\
\hline Other items (net) & -256 & -288 & -821 & -835 & -738 & -726 & -707 & -791 & -749 & -811 & -861 \\
\hline Assets & 121 & 82 & 88 & 101 & 102 & 110 & 114 & 119 & 121 & 113 & 110 \\
\hline IMF net & 50 & 5 & 3 & 0 & 2 & 1 & -1 & 2 & 0 & 0 & 0 \\
\hline Intra-central bank account & 8 & 5 & 4 & 4 & 3 & 3 & 3 & 3 & 7 & 3 & 3 \\
\hline Fixed and other assets & 50 & 58 & 59 & 70 & 70 & 79 & 82 & 83 & 88 & 90 & 88 \\
\hline Contested claims & 0 & 0 & 0 & 0 & 0 & 0 & 0 & 0 & 0 & 0 & 0 \\
\hline Capitalized expense & 13 & 14 & 23 & 28 & 27 & 26 & 30 & 31 & 27 & 20 & 19 \\
\hline Liabilities & 378 & 370 & 909 & 937 & 840 & 835 & 821 & 910 & 870 & 923 & 971 \\
\hline Capital & 378 & 370 & 909 & 937 & 840 & 835 & 821 & 910 & 870 & 923 & 971 \\
\hline Capital and reserves & 272 & 254 & 776 & 794 & 697 & 703 & 683 & 757 & 714 & 777 & 812 \\
\hline Provisions & 21 & 28 & 29 & 29 & 32 & 41 & 41 & 42 & 42 & 53 & 53 \\
\hline SDRs allocation & 58 & 74 & 82 & 81 & 82 & 79 & 78 & 83 & 80 & 79 & 79 \\
\hline Income \& expense account & 27 & 14 & 22 & 32 & 29 & 12 & 19 & 27 & 35 & 14 & 26 \\
\hline Valuation adjustment : & 0 & 0 & 0 & 0 & 0 & 0 & 0 & 0 & 0 & 0 & 0 \\
\hline Government lending funds & 0 & 0 & 0 & 0 & 0 & 0 & 0 & 0 & 0 & 0 & 0 \\
\hline Interoffice accounts and others & 0 & 0 & 0 & 0 & 0 & 0 & 0 & 0 & 0 & 0 & 0 \\
\hline
\end{tabular}

Sources: Data provided by the Cambodian authorities.

1/ Effective May 1994, deposits required of new commercial banks prior to their commencing operations.

2/ Consists mainly of holdings of short-term securities issued by foreign governments.

3/ Related to an old loan from the Foreign Trade Bank of Cambodia (FTBC) to the National Bank of Cambodia for printing expenses. 
Table 19. Cambodia: Interest Rates, 1997-2000 1/

(Percent per annum)

\begin{tabular}{|c|c|c|c|c|c|c|c|c|c|c|c|c|c|c|}
\hline & \multicolumn{4}{|c|}{1997} & \multicolumn{4}{|c|}{1998} & \multicolumn{4}{|c|}{1999} & \multicolumn{2}{|l|}{2000} \\
\hline . & Mar. & Jun. & Sep. & Dec. & Mar. & Jun. & Sep. & Dec. & Mar & Jun & Sep & $\overline{\mathrm{Dec}}$ & Mar & June \\
\hline \multicolumn{15}{|l|}{ Deposit rates } \\
\hline Riel savings deposits & 8.5 & 8.8 & 7.4 & 7.4 & 7.9 & 7.9 & 7.9 & 7.5 & 7.7 & 7.2 & 7.1 & 7.3 & 7.3 & 7.2 \\
\hline Riel term deposits $2 /$ & 11.4 & 11.4 & 11.4 & 11.4 & 11.4 & 11.4 & 11.4 & 11.4 & 22.4 & 10.9 & 9.2 & 8.7 & 8.7 & 8.7 \\
\hline Foreign currency savings deposits & 2.5 & 2.5 & 2.3 & 2.4 & 2.5 & 2.5 & 2.5 & 2.5 & 2.6 & 2.4 & 2.3 & 2.2 & 2.2 & 2.2 \\
\hline Foreign currency term deposits & 3.8 & 3.8 & 3.7 & 3.8 & 4.0 & 4.1 & 4.1 & 3.9 & 4.0 & 3.9 & 3.6 & 3.5 & 3.5 & 3.5 \\
\hline \multicolumn{15}{|l|}{ Lending rates $3 /$} \\
\hline \multirow{2}{*}{\multicolumn{15}{|c|}{$\begin{array}{l}\text { Foreign currency loans: rates charged } \\
\text { to private enterprises }\end{array}$}} \\
\hline & 18.3 & 18.4 & & 18.4 & 18.5 & 18.5 & 18.6 & 17.7 & 17.7 & 17.8 & 17.5 & 17.3 & 17.1 & 17.3 \\
\hline
\end{tabular}

Source: Data provided by the Cambodian authorities

1/ Simple averages of rates reported by the ten commercial banks with the largest deposits.

$2 /$ The average rate shown is that reported for three-month riel deposits. The volume of total riel deposits continues to be small.

3/ Virtually all loans to the private sector in Cambodia are denominated in foreign currencies. 
Table 20. Cambodia: Prudential Regulations

(As of June 2000)

\begin{tabular}{|c|c|c|}
\hline Regulations & Main features & $\begin{array}{l}\text { Issue date of relevant } \\
\text { regulations } 1 /\end{array}$ \\
\hline Large exposure & $\begin{array}{l}\text { Banks are not allowed to grant credit to an individual customer exceeding } 20 \\
\text { percent of their net worth. }\end{array}$ & $\begin{array}{l}\text { Dec. } 29,1997 \\
\text { Feb. } 17,2000\end{array}$ \\
\hline Licensing & $\begin{array}{l}\text { All entities that conduct banking activities must be licensed by the NBC. The } \\
\text { NBC determines the conditions for licensing. }\end{array}$ & Oct. 25,1996 \\
\hline Liquidity & Banks must at all times have a liquidity ratio of at least 100 percent. & Feb. 9,2000 \\
\hline $\begin{array}{l}\text { Loan / deposit ratio } \\
\text { for banks and } \\
\text { financial institutions }\end{array}$ & $\begin{array}{l}\text { The total amount of domestic deposits must be greater than or equal to the } \\
\text { sum of domestic loans, deposits with other local banks and the NBC, and vault } \\
\text { cash. }\end{array}$ & Aug. 28,1998 \\
\hline $\begin{array}{l}\text { Maintenance of } \\
\text { required reserves } \\
\text { against deposits and } \\
\text { other borrowing }\end{array}$ & $\begin{array}{l}\text { Reserve requirements of } 8 \text { percent are levied on deposits in both foreign and } \\
\text { domestic currency. Reserves are defined as cash-in-vault or unremunerated } \\
\text { deposits at the NBC, are calculated on the basis of average daily deposit } \\
\text { liabilities over successive } 28 \text { day periods, and must be met on an average basis } \\
\text { over the period. Penalties for noncompliance are imposed by charging } 1 / 10 \text { of } \\
\text { the latest refinancing rate per day on the deficiency. }\end{array}$ & $\begin{array}{l}\text { Dec. } 30,1993 \\
\text { Jan. } 1,1998\end{array}$ \\
\hline Minimum capital & $\begin{array}{l}\text { Locally incorporated banks and foreign bank branches must have paid up } \\
\text { minimum capital equal to at least CR } 50 \text { billion. In addition, each bank must } \\
\text { maintain } 10 \text { percent of authorized capital in a special capital account at the } \\
\text { NBC, as a form of security. The first } 5 \text { percent is unremunerated. The second } \\
5 \text { percent is remunerated at a rate that follows SIBOR. }\end{array}$ & $\begin{array}{l}\text { Jan. } 1,1994 \\
\text { Jan. } 1,1998 \\
\text { Feb. } 9,2000\end{array}$ \\
\hline $\begin{array}{l}\text { Monitoring banks } \\
\text { open foreign } \\
\text { exchange positions }\end{array}$ & $\begin{array}{l}\text { Banks are required to observe a limit on the long or short position in each } \\
\text { currency not exceeding } 5 \text { percent of the net worth, and a limit on the aggregate } \\
\text { short position not exceeding } 15 \text { percent of the net worth. }\end{array}$ & Jan. 16, 1995 \\
\hline $\begin{array}{l}\text { Provisions for bad } \\
\text { and doubtful debts }\end{array}$ & $\begin{array}{l}\text { Banks are required to classify their assets and off-balance sheet commitments } \\
\text { into the four classifications (standard, substandard, doubtful, and lost), and the } \\
\text { specific provisioning shall be recorded depending on the classifications in the } \\
\text { account and charged to the profit and loss account in the month identified. }\end{array}$ & $\begin{array}{l}\text { Dec. } 31,1994 \\
\text { Feb. } 17,2000\end{array}$ \\
\hline $\begin{array}{l}\text { Solvency ratio } \\
\text { (capital adequacy) }\end{array}$ & $\begin{array}{l}\text { Banks must maintain solvency ratio (net worth divided by the aggregate of } \\
\text { assets and off balance sheet commitments) at the minimum level of } 20 \text { percent. }\end{array}$ & $\begin{array}{l}\text { May } 22,1995 \\
\text { Feb. } 16,2000\end{array}$ \\
\hline $\begin{array}{l}\text { Other operational } \\
\text { regulations for banks } \\
\text { and financial } \\
\text { institutions }\end{array}$ & $\begin{array}{l}\text { Banks must maintain their net worth at least equal to their paid-up capital. } \\
\text { Banks are not allowed to grant credit to their own shareholders, members of } \\
\text { board of directors, managers, individuals or legal entities who participate in } \\
\text { their establishments. } \\
\text { Banks are not allowed to use demand deposits to grant long-term credit. } \\
\text { Banks are not allowed to acquire fixed assets except for operating purposes. } \\
\text { The total amount of fixed assets must not exceed } 30 \text { percent of net worth. }\end{array}$ & Dec. 29,1997 \\
\hline
\end{tabular}

Source: Data provided by the Cambodian authorities.

1/ Includes regulations, prakas (announcements), and circulars. 
Table 21. Cambodia: Balance of Paýments, 1995-99

(In millions of U.S. dollars; unless otherwise indicated)

\begin{tabular}{|c|c|c|c|c|c|}
\hline & 1995 & 1996 & 1997 & 1998 & $\begin{array}{r}1999 \\
\text { Est. }\end{array}$ \\
\hline Current account excluding official transfers & -513 & -493 & -252 & -230 & -346 \\
\hline Current account including official transfers & -167 & -193 & -43 & -36 & -126 \\
\hline Trade balance & -446 & -476 & -263 & -193 & .294 \\
\hline Exports & 798 & 618 & 786 & 854 & 933 \\
\hline Domestic exports $1 /$ & 268 & 295 & 534 & 610 & 695 \\
\hline Re-exports & 530 & 322 & 252 & 245 & 238 \\
\hline Imports, f.o.b. & -1244 & -1093 & -1050 & -1048 & -1227 \\
\hline Of which: retained imports, f.o.b. & -714 & -771 & -798 & -803 & -990 \\
\hline Services (net) & -97 & -63 & -49 & -97 & -122 \\
\hline Receipts & 129 & 198 & 205 & 171 & 202 \\
\hline Of which: tourism & 53 & 82 & 68 & 45 & 63 \\
\hline Payments & -226 & -261 & -254 & -268 & -324 \\
\hline Of which: interest & -11 & -14 & -17 & -18 & -17 \\
\hline Private transfers & 31 & 46 & 60 & 60 & 70 \\
\hline Official transfers & 346 & 299 & 210 & 194 & 220 \\
\hline Of which: budget support & 21 & 14 & 5 & 0 & 0 \\
\hline Of which: project aid & 154 & 156 & 110 & 105 & 150 \\
\hline Capital secount & 88 & 123 & -47 & -69 & 28 \\
\hline Medium- and long-term loans & -40 & -56 & -81 & -69 & -68 \\
\hline Disbursements & 79 & 75 & 35 & 46 & 52 \\
\hline Amortization & -119 & -131 & -115 & -115 & -121 \\
\hline Foreign direct investment & 151 & 294 & 168 & 120 & 120 \\
\hline Short-term flows and errors and omissions $2 /$ & -23 & -115 & -135 & -120 & .24 \\
\hline Overall balance & -79 & -70 & -90 & -105 & -98 \\
\hline Financing & 79 & 70 & 90 & 105 & 98 \\
\hline Change in gross official reserves & .82 & -52 & -28 & -11 & -32 \\
\hline Debt rescheduling & 27 & 0 & 0 & 0 & 0 \\
\hline Change in arrears $(-=$ reduction $)$ & 91 & 122 & 118 & 117 & 122 \\
\hline Use of Fund credit & 42 & $\mathbf{0}$ & 0 & -1 & 8 \\
\hline Purchases/disbursements & 42 & 0 & 0 & 0 & 11 \\
\hline Repurchases/repayments & 0 & 0 & 0 & 1 & 3 \\
\hline \multicolumn{6}{|l|}{ Memorandum items: } \\
\hline \multicolumn{6}{|l|}{ Current account balance } \\
\hline Excluding official transfers (in percent of GDP) & -16.7 & -15.5 & -8.1 & -8.2 & -11.5 \\
\hline Including official transfers (in percent of GDP) & -5.4 & -6.1 & -1.4 & -1.3 & -4.2 \\
\hline Gross official reserves $3 /$ & 182 & 234 & 262 & 390 & 422 \\
\hline In months of imports of goods and services & 1.5 & 2.1 & 2.4 & 3.6 & 3.3 \\
\hline Net international reserves & 110 & 164 & 197 & 323 & 349 \\
\hline External debt 4 / (in percent of GDP) & 18.2 & 19 & 66 & 75 & 71 \\
\hline
\end{tabular}

Sources: Data provided by the Cambodian authorities; and Fund staff estimates.

1/ Includes estimates for unrecorded forestry exports.

2/ Believed to be primarily short-term capital and unrecorded imports as counterpart to forestry exports.

3/ Includes \$117 million associated with the return of Cambodian gold holdings by the BIS in 1998.

4/ Starting in 1997, includes $\$ 1,346$ million owed to countries of the former Council of Mutual Economic Assistance.

This amount is indicative and subject to negotiations and rescheduling. 
Table 22. Cambodia: Merchandise Exports, 1995-99

(In millions of U.S. dollars)

\begin{tabular}{|c|c|c|c|c|c|c|}
\hline & Unit & 1995 & 1996 & 1997 & 1998 & $\begin{array}{l}1999 \\
\text { Est. }\end{array}$ \\
\hline Total exports & & 798 & 618 & 786 & 854 & 933 \\
\hline Domestic exports & & 268 & 295 & 534 & 610 & 695 \\
\hline Garment export & & 28 & 102 & 279 & 392 & 536 \\
\hline Logs and sawn & & 185 & 148 & 224 & 178 & 105 \\
\hline Rubber $1 /$ & & 41 & 32 & 23 & 33 & 30 \\
\hline Volume & $(000 \mathrm{MT})$ & 40 & 32 & 28 & 57 & 58 \\
\hline Unit price & $(\$ / M T)$ & 1,026 & 985 & 799 & 575 & 513 \\
\hline Rice & & $\ldots$ & 1 & 1 & 1 & 5 \\
\hline Volume & $(000 \mathrm{MT})$ & $\ldots$ & 4 & 5 & 5 & 30 \\
\hline Unit price & $(\$ / M T)$ & $\ldots$ & 271 & 243 & 204 & 166 \\
\hline Fish & & 2 & 3 & 3 & 3 & 4 \\
\hline Volume & (MT) & 1 & 2 & 2 & 1 & 4 \\
\hline Unit price & $(\$ / M T)$ & 1,976 & 1,862 & 1,611 & 1,925 & 1,141 \\
\hline Others & & 3 & 2 & 4 & 3 & 10 \\
\hline Re-exports & & 530 & 322 & 252 . & 245 & 238 \\
\hline
\end{tabular}

Sources: Data provided by the authorities; and Fund staff estimates.

1/ Includes estimates for illegal exports. 
Table 23. Cambodia: Merchandise Imports, 1995-99

(In millions of U.S. dollars)

\begin{tabular}{|c|c|c|c|c|c|}
\hline & 1995 & 1996 & 1997 & 1998 & $\begin{array}{r}1999 \\
\text { Est. }\end{array}$ \\
\hline Total imports, f.o.b. $1 /$ & 1,244 & 1,093 & 1,050 & 1,048 & 1,227 \\
\hline Total imports, c.i.f. & 1,352 & 1,188 & 1,141 & 1,139 & 1,334 \\
\hline Freight \& insurance on imports & 108 & 95 & 91 & 91 & 107 \\
\hline Taxable imports & 955 & 739 & 740 & 553 & 744 \\
\hline Cigarettes & 193 & 209 & 188 & 144 & 119 \\
\hline Motorcycles & 36 & 18 & 17 & 44 & 36 \\
\hline Beer & 15 & 14 & 5 & 3 & 2 \\
\hline VCRs & 15 & 6 & 1 & 2 & 1 \\
\hline TV sets & 17 & 13 & 6 & 5 & 6 \\
\hline Audio cassettes & 4 & 3 & 2 & 2 & 3 \\
\hline Gold & 306 & 41 & 136 & 3 & 28 \\
\hline Vehicles & 22 & 25 & 26 & 15 & 27 \\
\hline Construction materials & 19 & 20 & 19 & 8 & 13 \\
\hline Clothing & 17 & 21 & 18 & 22 & 33 \\
\hline Cloth & 13 & 10 & 4 & 4 & 4 \\
\hline Petroleum products & 100 & 128 & 91 & 111 & 153 \\
\hline Sugar & 6 & 13 & 15 & 14 & 22 \\
\hline Cement & 12 & 15 & 16 & 13 & 20 \\
\hline Steel & 4 & 5 & 1 & 1 & 19 \\
\hline Other & 153 & 173 & 176 & 141 & 233 \\
\hline
\end{tabular}

Sources: Data provided by the Cambodian authorities; and Fund staff estimates.

1/ Includes imports for re-exports. 
Table 24. Cambodia: Investment Approvals by Sector, 1995-99

(Total fixed assets approved, in millions of US dollars)

\begin{tabular}{|c|c|c|c|c|c|}
\hline & 1995 & 1996 & 1997 & $1998^{\circ}$ & 1999 \\
\hline Total & 2,240 & 762 & 744 & 853 & 456 \\
\hline Agriculture & 5 & 92 & 61 & 50 & 10 \\
\hline Industries & 301 & 438 & 516 & 652 & 226 \\
\hline Agro Industry & 1 & 26 & 4 & 2 & .30 \\
\hline Building Material & 1 & 16 & 9 & 1 & 0 \\
\hline Cement & 136 & 7 & 205 & 54 & 6 \\
\hline Energy & 40 & 1 & 80 & 17 & 0 \\
\hline Food Processing & 38 & 30 & 6 & 9 & 12 \\
\hline Garment & 30 & 46 & 97 & 127 & 75 \\
\hline Petroleum & 25 & 22 & 32 & 1 & 1 \\
\hline Tobacco & 4 & 22 & 2 & 7 & 0 \\
\hline Wood Processing & 5 & 207 & 47 & 179 & 14 \\
\hline Others & 20 & 61 & 34 & 255 & 87 \\
\hline Services & 425 & 112 & 125 & 39 & 196 \\
\hline Construction & 197 & 28 & 1 & 3 & 18 \\
\hline Education & 98 & 0 & 0 & 2 & 0 \\
\hline Infrastructure & 117 & 0 & $2 \mathrm{I}$ & 10 & 0 \\
\hline Teleconmunication & 6 & 33 & 53 & 0 & 19 \\
\hline Transporation & 4 & 10 & 0 & 11 & 5 \\
\hline Others & 2 & 42 & 50 & 14 & 154 \\
\hline Tourism & 1,510 & 119 & 42 & 112 & 25 \\
\hline Hotel & 140 & 101 & 40 & 106 & 25 \\
\hline Tourism Centre & 1,370 & 7 & 1 & 6 & 0 \\
\hline Others & 0 & 11 & 0 & 0 & 0 \\
\hline
\end{tabular}

Source: The Ministry of Economy and Finance. 
Table 25. Cambodia: Investment Approvals by Investor Country of Origin, 1995-99

(Total fixed assets approved, in millions of US dollars)

\begin{tabular}{|c|c|c|c|c|c|}
\hline & 1995 & 1996 & 1997 & $1998^{\circ}$ & 1999 \\
\hline Total & 2,240 & 762 & 744 & 853 & 456 \\
\hline Cambodia & 332 & 144 & 166 & 296 & 260 \\
\hline Foreign & 1,908 & 618 & 578 & 557 & 196 \\
\hline America & 148 & 8 & 97 & 11 & .20 \\
\hline United States & 109 & 5 & 86 & 5 & 20 \\
\hline Canada & 39 & 3 & 11 & 6 & 0 \\
\hline Asia & 1,560 & 533 & 437 & 534 & 171 \\
\hline Thailand & 19 & 52 & 27 & 33 & 21 \\
\hline Malaysia & 1,411 & 194 & 66 & 124 & 14 \\
\hline Singapore & 104 & 33 & 15 & 21 & 1 \\
\hline Indonesia & 1 & 13 & 1 & 6 & 1 \\
\hline Vietnam & 0 & 0 & 0 & 0 & 1 \\
\hline Taiwan & 9 & 164 & 44 & 144 & 55 \\
\hline China & 3 & 37 & 36 & 108 & 46 \\
\hline Hong Kong & 12 & 24 & 69 & 91 & 30 \\
\hline Korea & 1 & 5 & 178 & 5 & 0 \\
\hline Japan & 1 & 11 & 0 & 1 & 2 \\
\hline Europe & 199 & 55 & 8 & 8 & 4 \\
\hline United Kingdom & 23 & 39 & 6 & 0 & 2 \\
\hline France & 175 & 10 & 1 & 1 & l \\
\hline Switzerland & 0 & 6 & 0 & 5 & 1 \\
\hline Portugal & 1 & 0 & 1 & 1 & 0 \\
\hline Others & 2 & 22 & 36 & 4 & 1 \\
\hline
\end{tabular}

Source: The Ministry of Economy and Finance. 
Table 26. Cambodia: Foreign Debt, 1995-99

(In millions of U.S. dollars)

\begin{tabular}{|c|c|c|c|c|c|}
\hline & 1995 & 1996 & 1997 & 1998 & $\begin{array}{r}1999 \\
\text { Est. }\end{array}$ \\
\hline Total external debt outstanding $1 /$ & 561 & 617 & 2,050 & 2,110 & 2,136 \\
\hline Multilateral & 187 & 258 & 287 & 348 & 387 \\
\hline World Bank & 60 & 103 & 126 & 156 & 180 \\
\hline $\mathrm{AsDB}$ & 53 & 85 & 95 & 128 & 146 \\
\hline $\mathrm{MMF}$ & 74 & 70 & 66 & 64 & 61 \\
\hline Bilateral & 375 & 358 & 1,763 & 1,762 & 1,749 \\
\hline New debt & 25 & 24 & 29 & 30 & 31 \\
\hline Rescheduled debt & 349 & 334 & 1,734 & 1,732 & 1,718 \\
\hline Total disbursements & 121 & 75 & 41 & 46 & 52 \\
\hline Multilateral & 104 & 75 & 39 & 45 & 50 \\
\hline World Bank & 26 & 43 & 28 & 15 & 24 \\
\hline $\mathrm{AsDB}$ & 36 & 32 & 11 & 30 & 26 \\
\hline IMF & 42 & 0 & 0 & 0 & 0 \\
\hline Bilateral & 17 & 0 & 2 & 1 & 2 \\
\hline New debt & 17 & 0 & 2 & 1 & 2 \\
\hline Rescheduled debt & 0 & 0 & 0 & 0 & 0 \\
\hline Total amortization & 119 & 131 & 115 & 116 & 118 \\
\hline Multilateral & 0 & 0 & 0 & 1 & 3 \\
\hline World Bank & 0 & 0 & 0 & 0 & 0 \\
\hline AsDB & 0 & 0 & 0 & 0 & 0 \\
\hline IMF & 0 & 0 & 0 & 1 & 3 \\
\hline Bilateral & 119 & 131 & 115 & 115 & 115 \\
\hline New debt & 4 & 1 & 1 & 1 & 1 \\
\hline Rescheduled debt & 0 & 16 & 0 & 0 & 0 \\
\hline Nonrescheduled debt & 6 & 6 & 6 & 6 & 6 \\
\hline Total interest & 6 & 7 & 8 & 8 & 7 \\
\hline Multilateral & 1 & 2 & 3 & 3 & 2 \\
\hline World Bank & 0 & 1 & 1 & 1 & 1 \\
\hline AsDB & 0 & 0 & 1 & 1 & 1 \\
\hline $\mathrm{IMF}$ & 1 & 1 & 1 & 1 & 0 \\
\hline Bilateral & 5 & 5 & 5 & 5 & 5 \\
\hline New debt & 0 & 1 & 1 & 1 & 1 \\
\hline Rescheduled debt & 1 & 1 & 1 & 1 & 1 \\
\hline Nonrescheduled debt & 4 & 3 & 3 & 3 & 3 \\
\hline
\end{tabular}

Sources: Data provided by the Cambodian authorities; and Fund staff estimates.

1/ Starting in 1997, includes $\$ 1,346$ million owed to countries of the former Council of Mutual Economic Assistance. This amount is indicative and subject to negotiations and rescheduling. 
Table 27. Cambodia: Status of State-Owned Enterprises

(As of June 2000)

\begin{tabular}{|c|c|c|c|c|c|c|c|c|c|c|c|c|}
\hline \multirow[b]{2}{*}{ Controlling Ministry } & \multicolumn{4}{|c|}{ - Enterprises to be retained } & \multicolumn{4}{|c|}{ Enterprises to be privatized } & \multicolumn{4}{|c|}{ Enterprises already privatized } \\
\hline & $\begin{array}{l}\text { Fully } \\
\text { active }\end{array}$ & $\begin{array}{c}\text { Reduced } \\
\text { activity }\end{array}$ & Idle & Total & $\begin{array}{l}\text { Fully } \\
\text { active }\end{array}$ & $\begin{array}{l}\text { Reduced } \\
\text { activity }\end{array}$ & Idle & Totai & $\begin{array}{c}\text { Leased } \\
\text { enterprises }\end{array}$ & $\begin{array}{c}\text { Joint } \\
\text { ventures }\end{array}$ & $\begin{array}{l}\text { Sold to } \\
\text { private sector }\end{array}$ & Total \\
\hline Agriculture & $\cdots$ & $\ldots$ & $\ldots$ & 0 & 13 & 2 & $\cdots$ & 15 & 22 & 2 & $\ldots$ & 24 \\
\hline Commerce & $\cdots$ & $\cdots$ & $\ldots$ & 0 & $\cdots$ & 2 & $\ldots$ & 2 & 15 & $\ldots$ & 5 & 20 \\
\hline Council of Ministers & 1 & $\ldots$ & $\ldots$ & 1 & $\ldots$ & $\cdots$ & $\ldots$ & 0 & 3 & 1 & 2 & 6 \\
\hline Culture & $\cdots$ & ... & $\cdots$ & 0 & $\ldots$ & $\ldots$ & $\ldots$ & 0 & 5 & $\ldots$ & $\ldots$ & 5 \\
\hline Defense & $\ldots$ & $\cdots$ & $\cdots$ & 0 & $\cdots$ & $\cdots$ & $\ldots$ & 0 & 14 & $\ldots$ & $\ldots$ & 14 \\
\hline Education & $\cdots$ & $\ldots$ & $\ldots$ & 0 & $\ldots$ & $\ldots$ & $\ldots$ & 0 & 4 & $\ldots$ & $\ldots$ & 4 \\
\hline Finance & $\ldots$ & $\ldots$ & $\ldots$ & 0 & $\cdots$ & $\cdots$ & $\ldots$ & 0 & $\cdots$ & $\ldots$ & 3 & 3 \\
\hline Foreign Affair & $\cdots$ & $\cdots$ & $\ldots$ & 0 & $\cdots$ & $\ldots$ & $\ldots$ & 0 & 8 & $\ldots$ & $\ldots$ & 8 \\
\hline Health & $\cdots$ & $\cdots$ & $\ldots$ & 0 & $\cdots$ & $\ldots$ & $\ldots$ & 0 & 1 & 1 & 1. & 3 \\
\hline Industry & $\cdots$ & $\ldots$ & $\ldots$ & 0 & $\ldots$ & 2 & $\ldots$ & 2 & 51 & $\ldots$ & 4. & 55 \\
\hline Information & $\cdots$ & $\ldots$ & $\cdots$ & 0 & $\cdots$ & $\cdots$ & $\cdots$ & 0 & 4 & $\ldots$ & $\ldots$ & 4 \\
\hline Interior & ... & $\ldots$ & $\ldots$ & 0 & $\ldots$ & $\ldots$ & $\ldots$ & 0 & 2 & $\ldots$ & 2 & 4 \\
\hline Planning & $\ldots$ & $\ldots$ & $\ldots$ & 0 & $\ldots$ & $\ldots$ & $\ldots$ & 0 & $\cdots$ & $\ldots$ & 1 & 1 \\
\hline Public works and transport & $\cdots$ & 1 & $\ldots$ & 1 & $\ldots$ & 4 & $\ldots$ & 4 & 15 & $\ldots$ & 3 & 18 \\
\hline Post and telecommunications & $\ldots$ & $\cdots$ & $\ldots$ & 0 & $\cdots$ & $\cdots$ & $\ldots$ & 0 & 2 & 6 & $\ldots$ & 8 \\
\hline State secretariat of civil aviation & $\cdots$ & $\cdots$ & $\ldots$ & 0 & $\ldots$ & $\ldots$ & $\ldots$ & 0 & 11 & $\ldots$ & $\ldots$ & 11 \\
\hline Tourism & $\ldots$ & ... & $\ldots$ & 0 & $\ldots$ & $\ldots$ & $\ldots$ & 0 & 6 & $\ldots$ & $\ldots$ & 6 \\
\hline Urbanization & $\cdots$ & $\ldots$ & $\ldots$ & 0 & $\ldots$ & 2 & $\ldots$ & 2 & 5 & $\ldots$ & $\ldots$ & 5 \\
\hline Phnom Penh Municipality & ... & $\cdots$ & $\cdots$ & 0 & $\cdots$ & $\cdots$ & $\cdots$ & 0 & 54 & $\cdots$ & 1 & 55 \\
\hline Total & 1 & 1 & 0 & 2 & 13 & 12 & 0 & 25 & 222 & 10 & 22 & 254 \\
\hline
\end{tabular}


Table 28. Cambodia: Previously State-Owned Enterprises and Assets Sold to the Private Sector

(As of June 2000)

\begin{tabular}{|c|c|c|c|c|}
\hline Business & Ministry & $\begin{array}{c}\text { Buyer's } \\
\text { nationality }\end{array}$ & Date of sale & $\begin{array}{l}\text { Sale price } \\
\text { (US\$) }\end{array}$ \\
\hline Total & & & & $26,794,876$ \\
\hline Flour Factory & Industry & $\ldots$ & July 24,1991 & 450,000 \\
\hline Soap Factory & Industry & Cambodia & October 25, 1991 & 85,800 \\
\hline Mechanical Factory $\$ 2$ & Industry & $\ldots$ & November 14,1991 & 360,000 \\
\hline Mechanical Factory \#4 & Industry & Cambodia & November 14,1991 & 225,000 \\
\hline Battery Shop & Industry & $\ldots$ & November 14, 1991 & 156,000 \\
\hline Battery Manufacturer & Industry & $\ldots$ & November 14, $1996^{\circ}$ & 80,000 \\
\hline Tire Shop & Industry & Cambodia & November 14, 1991 & 40,000 \\
\hline Mechanical Factory \#3 & Industry & $\ldots$ & November 15, 1991 & 440,000 \\
\hline Mechanical Factory $\# 9$ & Industry & $\ldots$ & November 15, 1991 & 420,000 \\
\hline Mechanical Factory \#7 & Industry & $\ldots$ & November 29, 1991 & 155,000 \\
\hline KM 6 Store & Industry & Cambodia & July 30,1992 & 56,000 \\
\hline Rubber Warehouse & Industry & Cambodia & July 30, 1992 & 25,000 \\
\hline Bata Shoe Factory & Industry & $\ldots$ & August 24, 1992 & 140,000 \\
\hline Soap Factory & Industry & Cambodia & August 24, 1992 & 72,000 \\
\hline Kbal Thnal Store & Industry & Cambodia & December 8, 1992 & 170,000 \\
\hline Print Shop & Culture & Cambodia & June 15,1993 & $\ldots$ \\
\hline Agriculture Material Manufacturer & Industry & Cambodia & June 17,1994 & ... \\
\hline Print Shop & Culture & Cambodia & June 17,1995 & $\ldots$ \\
\hline Tobacco Manufacturer & Industry & Cambodia & January 26,1996 & $3,400,000$ \\
\hline $\mathrm{CKC}$ & Commerce & Cambodia & March 11, 1996 & $10,680,000$ \\
\hline SOKLAIT (Warehouse and plant) & Industry & Cambodia & October 21,1996 & $1,250,000$ \\
\hline Bran Oil Factory & Industry & Cambodia & December 4, 1997 & $1,020,000$ \\
\hline Zeam Conte Garages 1 & Planing & Cambodia & October 24,1997 & 200,000 \\
\hline Zeam Conte Garages 2 & Commerce & Cambodia & January 27,1997 & 200,000 \\
\hline Gas Station in Seam Reap Province & Finance & Cambodia & October 8, 1997 & 40,000 \\
\hline Taprum Hotel & Finance & Cambodia & March 13, 1997 & 70,000 \\
\hline Peace Hotel & Finance & Cambodia & July 30,1998 & 40,000 \\
\hline Phnom Kamchay Hotel & Interior & Cambodia & June 15,1998 & 155,516 \\
\hline Vacant Land in Kampong spey Province & Interior & Cambodia & April 30, 1998 & 71,160 \\
\hline Pailin Hotel in Batambang Province & Commerce & Cambodia & February 18, 1998 & 45,666 \\
\hline Warehouse in Posat Province & Commerce & Cambodia & April 2, 1998 & 21,980 \\
\hline C.K.C. & Commerce & Cambodia & 1997 & 10,860 \\
\hline Focker Air Plane & Council of Ministers & Cambodia & November -98 & 663,970 \\
\hline Warehouse of Crop Company Group & Cornmerce & Cambodia & 1998 & 63,600 \\
\hline Chinese Hospital & Health & Cambodia & 1998 & $2,345,324$ \\
\hline Vacant Land near the Cigarette Factory & Industry & Cambodia & 1997 & 132,000 \\
\hline Beng Trabek Garage & Industry & Cambodia & January 26,1998 & $1,185,000$ \\
\hline Beverage Factory & Industry & Cambodia & July 20,1998 & 630,000 \\
\hline Warehouse of Acid Water & Public Works & Cambodia & July 21,1998 & 352,000 \\
\hline Vacant Land behind Council of Ministers & Council of Ministers & Cambadia & 1998 & 800,000 \\
\hline KAMTRANSHIP & Public Works & Cambodia & May 18,1998 & 543,000 \\
\hline Driving School Department & Public Works & Cambodia & December 5, 1999 & 250,000 \\
\hline Vacant Land in Phnom Penh Health Dept. & Phnom Penh Municipality & Cambodia & April 18, 2000 & 41,198 \\
\hline
\end{tabular}

Source: The Ministry of Economy and Finance. 
Table 29. Cambodia: State-Owned Enterprises Leased to the Private Sector

(As of June 2000)

\begin{tabular}{lcccc}
\hline & Number of & & Duration of lease & \\
\cline { 4 - 5 } Type of asset & leases & 1-14 years & $15-20$ years & $>20$ years \\
\hline & & & & 126 \\
Total & 222 & 22 & 74 & 0 \\
Beverage companies & 2 & 0 & 2 & 6 \\
Brick, ceramics, stone manufacturers & 7 & 0 & 1 & 3 \\
Car repair, ship repair & 4 & 1 & 0 & 1 \\
Chemical plants & 4 & 0 & 3 & 8 \\
Garages, gas stations & 16 & 0 & 8 & 9 \\
Land, buildings & 52 & 8 & 14 & 1 \\
Leather, textiles manufacturers & 19 & 0 & 10 & 20 \\
Mechanical plants & 4 & 0 & 3 & 15 \\
Miscellaneous manufacturing & 27 & 1 & 6 & 2 \\
Restaurants, guest house & 22 & 1 & 6 & 15 \\
Sawmills & 9 & 0 & 7 & 2 \\
Shops, offices & 26 & 11 & 0 & 14 \\
Tire factories & 6 & 0 & 4 & \\
Warehouses & 24 & 0 & 10 & \\
\hline
\end{tabular}

Source: The Ministry of Economy and Finance. 
Table 30. Cambodia: Proposed Privatization Decisions

(As of June 2000)

\begin{tabular}{|c|c|c|c|c|}
\hline \multirow{2}{*}{ Type of Enterprise } & \multirow{2}{*}{$\begin{array}{l}\text { Total Number } \\
\text { of Enterprises }\end{array}$} & \multicolumn{3}{|c|}{ Decisions } \\
\hline & & To be liquidated & To be privatized & To be retained \\
\hline Total & 27 & 13 & 12 & 2 \\
\hline Construction & 5 & 5 & $\ldots$ & $\ldots$ \\
\hline Ferry boat & 1 & $\ldots$ & $\ldots$ & 1 \\
\hline Fishery & 4 & 4 & $\ldots$ & $\ldots$ \\
\hline Goods transport & 5 & 2 & 3 & $\ldots$ \\
\hline Manufacturing & 1 & 1 & $\ldots$ & $\ldots$ \\
\hline Printing, video & 2 & 1 & $\ldots$ & 1 \\
\hline Rubber plantations & 7 & $\ldots$ & 7 & $\ldots$ \\
\hline Service industries & 1 & $\ldots$ & 1 & $\ldots$ \\
\hline Others & 1 & $\cdots$ & 1 & $\ldots$ \\
\hline
\end{tabular}

Source: The Ministry of Economy and Finance. 
Table 31. Cambodia: Joint Ventures Entered into by the State

(As of June 2000)

\begin{tabular}{|c|c|c|c|c|c|}
\hline \multirow[b]{2}{*}{ Company } & \multirow[b]{2}{*}{ Business } & \multirow[b]{2}{*}{ Ministry } & \multirow{2}{*}{$\begin{array}{c}\text { Share Retained by the } \\
\text { State in Percent }\end{array}$} & \multicolumn{2}{|c|}{ Private Interest } \\
\hline & & & & Nationality & Share in Percent \\
\hline CASOTIM & Wood Processing & Agriculture & 20 & Russia & 80 \\
\hline COLEXIM & Wood Processing & Agriculture & 61 & Japan & 49 \\
\hline Royal Air Cambodge & Airline & Council of Ministers & 60 & Malaysia & 40 \\
\hline Cambodia Samart Communication & Telecommunications & Post \& Telecommunications & 30 & Thailand & 70 \\
\hline Cambodia Shinawatra Co. & Telecommunications & Post \& Telecommunications & 30 & Thailand & 70 \\
\hline CAMTEL & Telecommunications & Post \& Telecommunications & 30 & Thailand & 70 \\
\hline SOCITEL & Telecommunications & Post \& Telecommunications & 30 & Thailand & 70 \\
\hline Telstra International Ltd. & Telecommunications & Post \& Telecommunications & 40 & Australia & 60 \\
\hline CAMINTEL & Telecommunications & Post \& Telecommunications & 51 & Indonesia & 49 \\
\hline Cambodia Pharmaceutical Enterprise & Medicine Processing & Health & 48 & China & 52 \\
\hline
\end{tabular}

Source: The Ministry of Economy and Finance. 
(as of December 1999)

\begin{tabular}{|c|c|c|c|}
\hline $\operatorname{Tax}$ & Nature of tax & Exemptions and deductions & Rates \\
\hline $\begin{array}{l}\text { 1. Taxes on income, } \\
\text { profits, and capital } \\
\text { gains } \\
\text { - Tax on salaries }\end{array}$ & $\begin{array}{l}\text { A monthly tax on income from salaries } \\
\text { (including remuneration, bonuses, overtime } \\
\text { compensations, and fringe benefits), } \\
\text { withheld at source. The tax applies to all } \\
\text { salaries received in Cambodia and to } \\
\text { salaries received abroad by Cambodian } \\
\text { nationals if the employer is a Cambodian } \\
\text { resident. }\end{array}$ & $\begin{array}{l}\text { Exempted from tax on salaries are: (i) salaries of diplomatic and other } \\
\text { foreign officials, such as international organization representatives, (ii) real } \\
\text { refunds on professional expenses, (iii) indemnity for the layoff provided } \\
\text { under the Labor Law, (iv) additional remuneration with social characteristics } \\
\text { provided under the Labor Law, (v) uniform and professional equipment } \\
\text { provided by employers, and (vi) travel expense allowance. } \\
\text { Deductions of CR } 75,000 \text { from the tax base per month are allowed for each } \\
\text { minor dependent child and a spouse without occupation. }\end{array}$ & $\begin{array}{l}\text { Progressive rates applied to monthly income are as follows: (i) CR } 0 \text {. } \\
500,000,0 \text { percent; (ii) CR } 500,001-1,250,000 \text { riel, } 5 \text { percent; (iii) CR } \\
1,250,001-8,500,000 \text { riel, } 10 \text { percent; (iv) CR } 8,500,001-12,500,000 \text { riel, } \\
15 \text { percent; and (v) CR } 12,500,000 \text { and over, } 20 \text { percent. }\end{array}$ \\
\hline - Tax on property rental & $\begin{array}{l}\text { A tax on receipts from rents on land and } \\
\text { buildings. The tax is paid by the owner. }\end{array}$ & $\begin{array}{l}\text { Property owners whose monthly income from rent is below } 500,000 \text { riel are } \\
\text { exempted. }\end{array}$ & 10 percent \\
\hline - Tax on profits & $\begin{array}{l}\text { A monthly tax on the profits of businesses, } \\
\text { including interest, rent and royalty received, } \\
\text { and capital gains from the sale of assets. } \\
\text { An enterprise under the real regime system } \\
\text { is subject to accounts-based profit taxes, } \\
\text { and an enterprise under the estimated } \\
\text { regime system is subject to a proxy profits } \\
\text { tax based on estimated tumover. }\end{array}$ & $\begin{array}{l}\text { Exemption from tax on profits provided under the Law on Taxation are: (i) } \\
\text { the income of the Royal Government and institutions of the Royal } \\
\text { Government, (ii) the income of religious, charitable, scientific, literary or } \\
\text { educational organization, (iii) the income of labor organization, chamber of } \\
\text { commerce, industry or agriculture, and (iv) the profit from the sale of } \\
\text { agricultural produce. In addition, under the Law on Investment, exemptions } \\
\text { can be granted by the Council for the Development of Cambodia (CDC) to } \\
\text { investing enterprises for up to } 8 \text { years, commencing the year the investment } \\
\text { project first becomes profitable. } \\
\text { Deductions allowed from tax on profits are: (I) interest expenses, (ii) } \\
\text { depreciation of tangible, and intangible property, and natural resources, and } \\
\text { (iii) charitable contributions. Cany forward of losses is also allowed. }\end{array}$ & $\begin{array}{l}\text { For corporation, the tax rates applied to annual profits are as follows; (i) an } \\
\text { investment enterprise during the period of tax exemption, } 0 \text { percent; (ii) and } \\
\text { investment enterprise after the period of tax exemption, } 9 \text { percent; (iii) } \\
\text { enterprises engage in production or exploitation of oil, gas, natural resources } \\
\text { including timber, ore, gold, and precious stones, } 30 \text { percent; (iv) other legal } \\
\text { person, } 20 \text { percent. } \\
\text { For non corporation, progressive rates applied to annual profits are as } \\
\text { follows: (i) CR } 0-6,000,000,0 \text { percent; (ii) CR } 6,000,001-15,000,000,5 \\
\text { percent; (iii) CR } 15,000,001-102,000,000 \text { riel, } 10 \text { percent; (iv) CR } \\
102,000,001-150,000,000 \text { riel, } 15 \text { percent; and (v) } 150,000,000 \text { and higher, } \\
20 \text { percent. }\end{array}$ \\
\hline 2. Patent tax & $\begin{array}{l}\text { An annual registration or license fee levied } \\
\text { on all businesses, industries, and services. } \\
\text { The base is the previous year's turnover, or } \\
\text { estimated tumover. New businesses are } \\
\text { taxed on the basis of a provisional estimate. }\end{array}$ & Farmers and business under the family kind are not subject to the tax. & 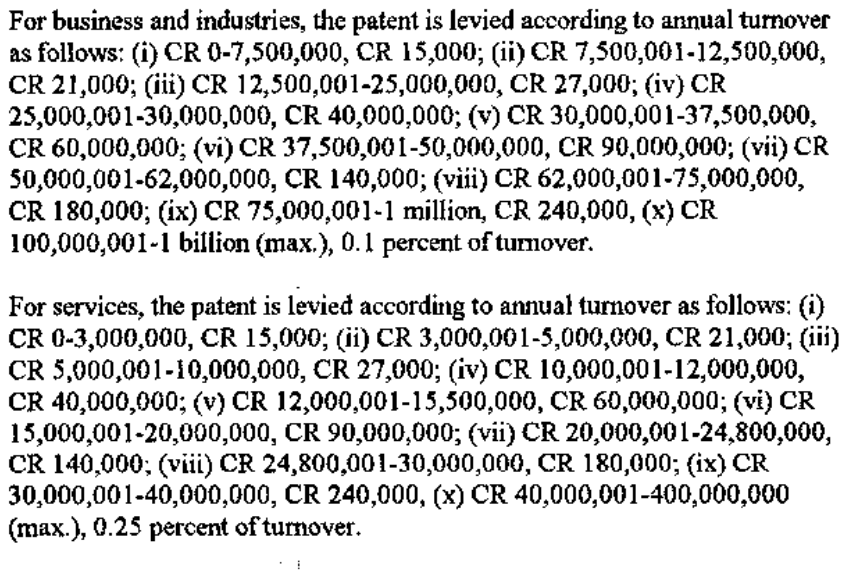 \\
\hline
\end{tabular}


(as of December 1999)

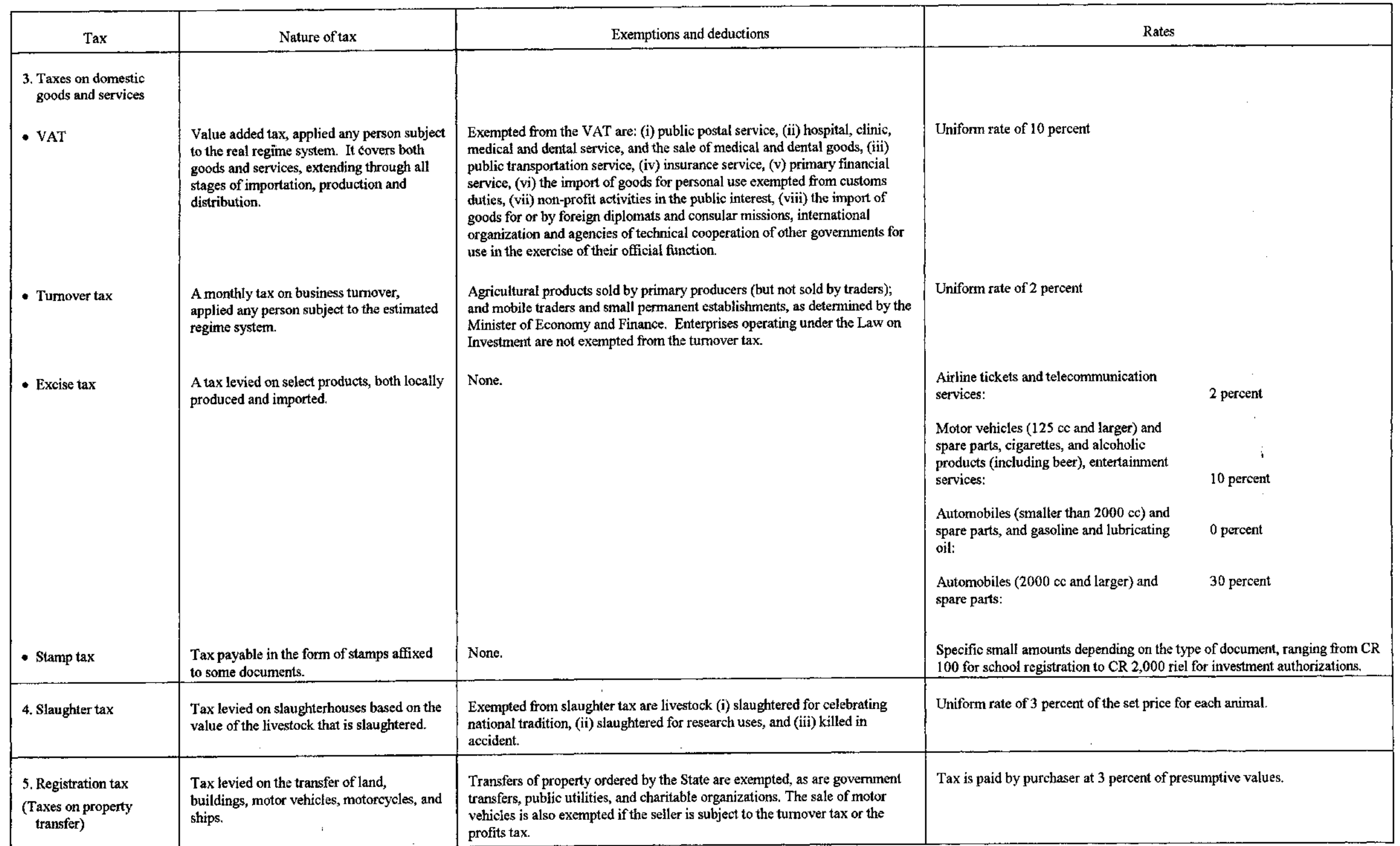


(as of December 1999)

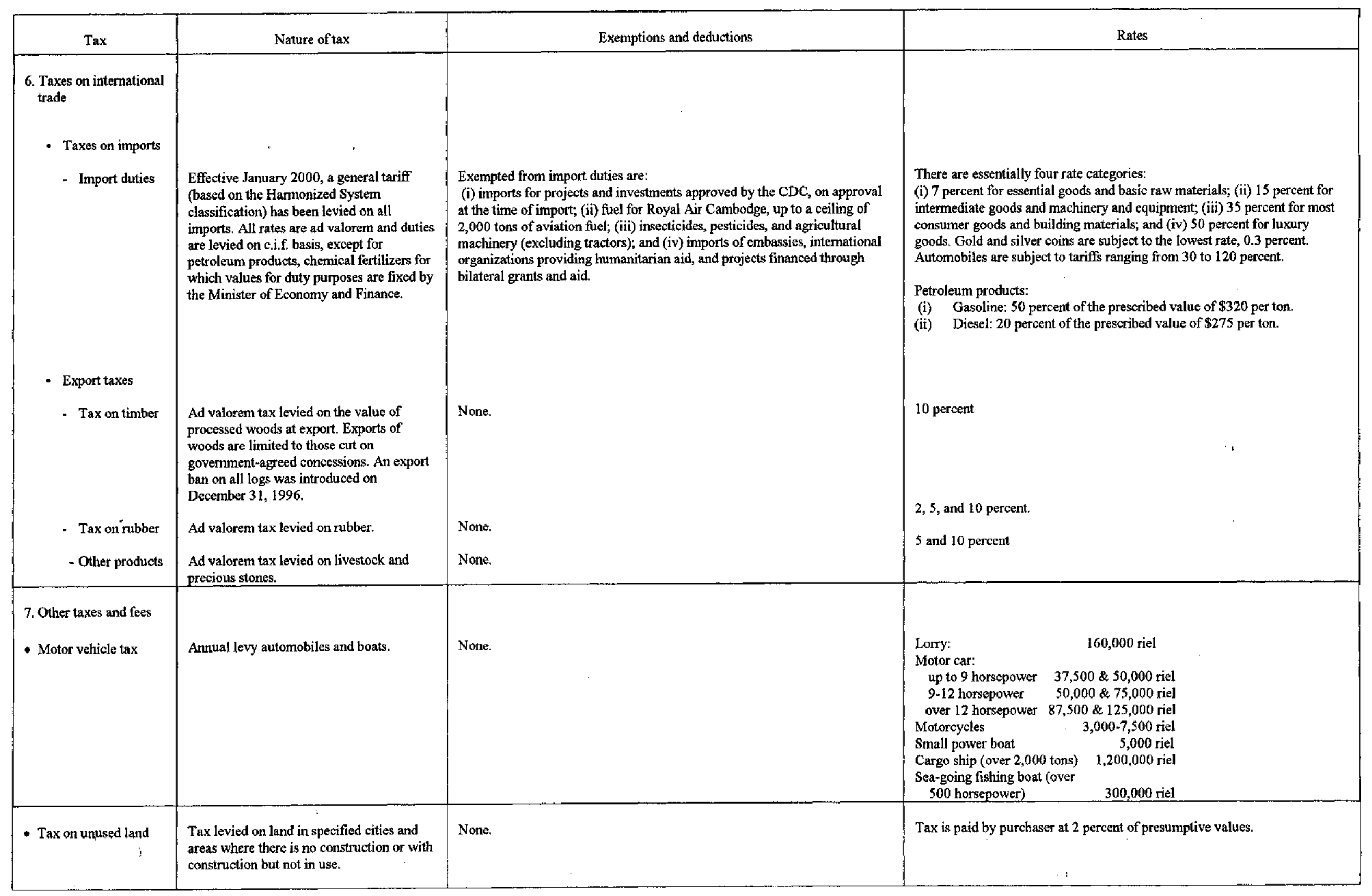

